\title{
Advertising and consumer search in a duopoly model $^{*}$
}

\author{
Maarten C.W. Janssen ${ }^{\dagger} \quad$ Marielle C. Non ${ }^{\ddagger}$
}

March 15, 2005

\begin{abstract}
We consider a duopoly in a homogenous goods market where part of the consumers are ex ante uninformed about prices. Information can come through two different channels: advertising and sequential consumer search. Despite the similarities in the models, our results differ substantially from those obtained by Robert and Stahl (1993). First, advertising and search are "substitutes" for a large range of parameters. Second, there is no monotone relationship between prices and the degree of advertising. Third, when the cost of either search or advertising vanishes, the competitive outcome arises. Finally, both expected advertised and non-advertised prices are non-monotonic in search cost. One of the implications is that firms actually may benefit from consumers having low (rather than high) search costs.
\end{abstract}

Keywords: consumer search, advertising, price dispersion JEL codes: D83, L13, M37

${ }^{*}$ We thank Jose Luis Moraga and other seminar participants at Tinbergen Institute Rotterdam for helpful comments and suggestions. We also thank Vladimir Karamychev for help in plotting the equilibrium regions.

${ }^{\dagger}$ Dept. of Economics, Erasmus University Rotterdam and Tinbergen Institute, P.O. Box 1738, 3000 DR Rotterdam, The Netherlands. E-mail: janssen@few.eur.nl.

${ }^{\ddagger}$ Corresponding author, Tinbergen Institute and dept. of Economics, Erasmus University Rotterdam, P.O. Box 1738, 3000 DR Rotterdam, The Netherlands. E-mail: non@few.eur.nl 


\section{Introduction}

Consumers and firms do not find each other costlessly in the marketplace. Consumers spend time, money and effort searching to find firms that offer the price and quality that best suits their tastes. Firms, on the other hand, spend money on trying to attract (new) consumers. It is true that the use of the internet has facilitated this process of reaching out for agents on the other side of the market. However, internet penetration (and certainly the use of search engines) is still quite low in many markets. Moreover, in those active electronic markets where the internet may have made it much cheaper for some consumers to search for the best offer, it is still the case that firms have to pay considerable sums of money for advertising.

There is a considerable amount of economic literature on both advertising (see, e.g., the seminal papers by Butters (1977)) and search (see, e.g., seminal papers by Diamond (1971), Burdett and Judd (1983) and Stahl (1989)). The separate study of firms' incentives to advertise or consumers' incentives to search and the implications of these incentives for the behavior of markets has yielded interesting insights. One important finding that can be consistently found throughout this large literature is that price dispersion may be consistent with equilibrium behavior. Still, if consumers for example do not have adequate incentives to bring about competitive behavior on the part of individual firms, one may ask the question whether in these cases of "insufficient" search, firms may have incentives to advertise their products. Conversely, if the literature finds that firms do not advertise their products enough, one may wonder whether consumers have incentives to search more. This interaction between firms' incentives to advertise and consumers' incentives to search is the core of this paper.

To the best of our knowledge there exist only two papers where the interaction between advertising and search activities is studied before. In their seminal paper Robert and Stahl (1993) study the equilibrium properties of a homogeneous goods model where in the first stage firms choose an advertising intensity and a price they charge for their product; after (not) having observed firms' advertisements, consumers decide in the second stage on whether or not to search for (more) prices in a sequential way, i.e., they first choose whether or not to obtain one price quotation and after having seen the result of this price search they decide whether to continue searching or not. All consumers are identical and have to pay a search cost $c$ per search. The advertising technology is convex, i.e., it requires more and more money to reach one more consumer. Despite the fact that they cannot get a closedform solution for the equilibrium strategies, Robert and Stahl reach a few important results. First, the equilibrium always exhibits price dispersion. Second, lower prices are more heavily advertised than higher prices. Third, there exists a complementarity between search and advertising in the sense that for those parameter values for which firms do not advertise, consumers 
do not search either (autarky). Moreover, whenever firms do advertise, consumers also are engaged in search activities. Fourth, there is an important asymmetry between advertising cost approaching zero and search cost approaching zero: in the first case market behavior converges to competitive pricing, whereas in the second case it does not. Stahl (2000) builds on the model by Robert and Stahl (1993) and analyzes some specific cases, the most important modification being that he has a different interpretation of the search cost parameter.

Our paper can be considered a two-firm version of the Robert and Stahl paper with two important modifications. First, we allow for the fact that consumers are heterogeneous in the sense that different consumers have different search costs: some consumers have a given positive search cost, whereas others have zero search cost either because they enjoy shopping or have a negligibly small opportunity cost of time or because they use search engines. This assumption often made in the search literature reflects the fact that search engines are used more and more in some market segments where internet purchases have some impact on overall market behavior. Second, we interpret the search cost parameter as the cost of searching a firm, while Robert and Stahl interpret the search cost as the cost of visiting a firm. In their model therefore consumers also have to pay search costs when buying from a firm they got an advertisement from, while in our model visiting an advertising firm is costless. In general, search costs consist of two components: the cost of visiting a shop knowing that the shop carries the product the consumer wants to buy and the cost of finding a shop that carries the product. An ad eliminates the importance of the second component of search costs. From this perspective, Robert and Stahl (1993) consider one extreme situation, namely one where the second component of search costs is absent. In this case the role of advertising is to inform consumers on the price and if this price is low enough compared to the expected price in other (non-advertising) shops the consumer buys from the advertising shop. However, this consumer then still has to pay visiting costs. We consider the other extreme situation, namely the one where the costs of visiting a shop are negligibly small compared to the costs of finding a shop that sells the product. The role of advertising here is twofold: an ad informs consumers that the shop sells the product and tells against which price. This makes consumers save on search costs. In fact, in this situation a firm in a sense pays the search costs for the consumer to gain a competitive advantage on non-advertising firms that have to be searched for by the consumer. Apart from these two modifications, we also consider a linear advertising technology where the cost of reaching an additional consumer is independent of the fraction of consumers already reached. ${ }^{1}$ This allows us to get closed-form

\footnotetext{
${ }^{1}$ There are some other stylistic modifications in presentation which turn out to be unimportant for explaining the considerable differences in results. We allow firms to
} 
solutions which make the analysis easier to understand.

Despite the fact that these assumptions seem to embody only minor modifications vis-a-vis the model of Robert and Stahl (1993), we show that our results differ considerably from theirs. First, in our model there is a considerable degree of substitutability between advertising and search: in case consumers for some reason economize on their search activities, firms tend to increase their advertising expenditures as long as advertising is not too expensive. On the other hand, when firms economize on advertising, consumers tend to search more as long as the search costs are not too high. Second, there is no consistent relationship between advertised and non-advertised prices. In particular, unlike the results obtained in Robert and Stahl (1993) it is not always the case that lower prices will be advertised more and higher prices will be advertised less. Third, expected prices are non-monotonic in changes in the search cost parameter, but when search costs approach zero, our equilibrium prices converge to the competitive price level. We will now briefly explain the main ideas behind our results and indicate why our results differ from those obtained by Robert and Stahl (1993). First, in our model advertising and search are 'substitutes'. This is partly caused by the existence of consumers that search for free, called shoppers. The existence of shoppers drives the prices down and in this way can make it attractive to search, even when the firms do not advertise. In Robert and Stahl's model, assuming shoppers do not exist, advertising is necessary to lower prices. Therefore, in this case, in an equilibrium where consumers search, firms need to advertise. Furthermore, the interpretation of the search cost in Robert and Stahl's model leads to the conclusion that an equilibrium where firms advertise without search is not possible. The main idea behind this result is that if consumers have to pay search costs when visiting an advertising firm, these firms need to lower their prices by at least as much as the search costs, leading non-advertising firms to lower their price too, in this way facilitating search. When the search costs are interpreted as the cost of searching a firm, like in our model, advertising firms can ask high prices, making search unprofitable.

In our model, advertised prices may be higher than non-advertised prices. There are two different cases where this can happen; when the advertising probability is low and when there is little consumer search. In the first case both advertising and non-advertising firms face the same competition on the shopper segment. However, an advertising firm knows almost for sure he will face no competition over the non-shoppers that receive its ad, while a non-advertising firm has monopoly power only on a part of the searching

randomize between not advertising and advertising with costs $A$. When firms do advertise they are assumed to reach all consumers. In equilibrium, firms may not advertise, advertise for sure, or advertise with a certain positive probability $\alpha$. It turns out that this can be reinterpreted as firms advertising with reach $\alpha$, and $\operatorname{costs} \alpha A$. See also footnote 5 for more details. 
non-shopper segment. Since an advertising firm has monopoly power over a larger fraction of consumers considering buying from it, it has a larger incentive to raise its price. In the second case, when consumer search is low, non-advertising firms sell virtually nothing to the non-shoppers. A nonadvertising firm therefore has to compete for the shoppers, leading to severe price competition and low prices. Both arguments do not hold in Robert and Stahl's model, since they don't allow for shoppers.

Another reason why in our model advertised prices can be higher than nonadvertised prices is the difference in interpretation of the search costs. In our model, receiving an ad means that a consumer does not have to pay search costs, and so advertised prices can be fairly high. In Robert and Stahl's model a consumer receiving an ad still has to pay search costs to visit the firm and so advertised prices should be lower than in our model. In fact, in our model the effect of advertising is two-sided: advertising attracts consumers that otherwise would have searched a competitor's shop and advertising 'pays' the search costs for these consumers. In Robert and Stahl's model advertising has only the effect that it attracts consumers that otherwise would have searched the competitor's firm.

To understand the reason why expected prices are non-monotonic with respect to changes in search cost, it is important to understand the reason why there is price dispersion in this model. Price dispersion arises from the fact that in equilibrium there are different types of consumers: those that are informed of all prices (or at least more than one firm's price) and those that have only observed one price (either through advertisement or through search). Over the last group of consumers, firms have monopoly power, but there is competition for the first group of consumers. Price dispersion is the way firms balance these two forces. When search cost decline, it is natural for consumers to search more ceteris paribus. This forces firms to lower their prices. On the other hand, when consumers search more, and with lower prices, firms have an incentive to lower their advertising intensity and thereby to increase prices (as ceteris paribus there are fewer consumers who make price comparisons). In some cases, the first tendency is larger than the second; in other cases, the second tendency is larger. ${ }^{2}$ When search costs are very small, firms don't advertise at all. This means that firms cannot decrease their advertising intensity further and the model behaves as a search model with a fraction of fully informed consumers. Prices converge to marginal cost in these models, when search costs become arbitrarily small. Another interesting aspect is the following. There is now some literature on the relation between consumer search costs and firm profits. For instance, Bakos (1997) argues that in homogenous goods markets firms have incentives

\footnotetext{
${ }^{2}$ Expected (non-advertised) prices are non-monotonic in advertising costs as well. The reason here is again that there are two effects, namely a decrease in advertising and an increase in advertised price, that work against each other. This analysis is not that simple however and depends on the type of equilibrium that exist. More on this in Section 4.
} 
to increase if possible search and switching costs to deter search. The idea here is, of course, that firms can charge higher prices and make more profits when consumers do not search a lot. This is also the starting point of Kuksov (2004). He, however, shows that with decreasing search costs firms also have incentives to differentiate, thereby lowering price competition. This can finally lead to higher profits when search costs decrease. In our model a same type of reasoning holds, although instead of allowing firms to change the product design, we allow firms to decrease their advertising activities, possibly leading to less competition and higher prices when search costs decrease.

The rest of the paper is organized as follows. Section 2 presents the model and Section 3 gives a full characterization of the equilibrium configurations possible. The most important comparative static results are presented in Section 4 and Section 5 concludes. The more lengthy proofs can be found in the Appendix.

\section{Model}

Our model deals with a homogeneous good market with two active firms. ${ }^{3}$ The production costs of the good are constant and equal across firms. We will normalize the production costs to 0 . There are no capacity constraints. Firms have the possibility to advertise. The per consumer advertising costs are $A$. Advertising is an 'all-or-nothing' decision, that is, a firm either does not advertise or it advertises to the complete market. In an ad the firm informs the consumers that it exists and sells the product and it mentions the price it charges. It is assumed firms have to stick to the price they announce in their ad, that is, ads never lie, and that they have to set a single price to all consumers.

At the demand side of the market there is a unit mass of consumers. Each consumer demands a single unit of the good and has valuation $\theta>0$ for the good. We will assume that $\theta>A$, otherwise it is clear firms will not advertise. A fraction $\gamma$, with $0<\gamma<0.5$, of the consumers is called shoppers. These consumers are assumed to know the prices charged by both firms and they will buy at the firm with the lowest price (provided this price is lower than $\theta$ ). The other $1-\gamma$ consumers a priori do not know the prices charged by the two firms. Sometimes they will get an ad from one or both firms, depending on the advertising strategy of the firms. Consumers can also decide to search one or both firms for prices. It is assumed that search is costly:

\footnotetext{
${ }^{3}$ An important part of the analysis of Robert and Stahl (1993) deals with the $N$ firms case as one question they are interested in is whether market outcomes get closer to the competitive outcomes when $N$ increases. As the model cannot be explicitly solved for $N>2$, we will not deal with this issue.
} 
each search action $\operatorname{costs} c$, where $c<\theta$. Note that when $c>\theta$, consumers will not search. Consumers have perfect memory; they know which firms they already searched and also remember which price they found there. Furthermore it is assumed that consumers receive all advertisements that are sent by the firms before they start to search. This implies that only nonadvertising firms will possibly be searched and that firms that have been searched will not be searched for a second time. It is assumed that search is sequential: after each search action the consumer decides whether or not to continue searching.

The timing of the game is as follows. First the firms simultaneously decide on their advertising and pricing strategies. With probability $\alpha^{j}$ a firm $j$ advertises and chooses a price from price distribution $F_{1}^{j}(p)$, where $F_{1}^{j}(p)$ denotes the probability that a price smaller than or equal to $p$ is chosen. ${ }^{4}$ With probability $1-\alpha^{j}$ a firm does not advertise and chooses a price from price distribution $F_{0}^{j}(p)$. So a firm $j$ 's strategy is given by $\left\{\alpha^{j}, F_{0}^{j}(p), F_{1}^{j}(p)\right\} .{ }^{5}$

After the firms have decided on their strategy, advertisements are realized according to $\alpha^{j}$ and prices are drawn from $F_{0}^{j}(p)$ or $F_{1}^{j}(p)$. We will denote by $\bar{p}_{0}^{j}$ and $\bar{p}_{1}^{j}$ the upper bounds of the supports ${ }^{6}$ of price distributions $F_{0}^{j}(p)$ and $F_{1}^{j}(p)$ respectively. In the same way, $\underline{p}_{0}^{j}$ and $\underline{p}_{1}^{j}$ denote the respective lower bounds. The shoppers now buy at the lowest-priced firm (provided the price is lower than $\theta$ ). The non-shoppers first see the advertisements and then decide on their search strategy. If they decide not to search, they buy at the firm with the lowest advertised price lower than $\theta$ (or, if there are no ads, they do not buy at all). If they decide to search they pick a non-advertising firm at random and obtain a price quotation from that firm. This price quotation is added to the set of already obtained price quotations, consisting of all advertised prices and the prices that have been

\footnotetext{
${ }^{4}$ We will see later that the unique symmetric equilibrium requires mixed strategies. Pure strategies are however also included in this specification: a firm that plays pure (price) strategy $\hat{p}$ has $F_{1}^{j}(p)=0$ for $p<\hat{p}$ and $F_{1}^{j}(p)=1$ for $p \geq \hat{p}$.

${ }^{5}$ It may seem that this way of modelling a firm's strategy is different from the one in Robert and Stahl (1993), where a firm's strategy is denoted by (in our notation) $\left\{p, \alpha^{j}(p)\right\}$ implying a firm can condition its advertising decision on the price it charges and not vice versa. It can be shown, however, that there is no fundamental difference between these two ways of modelling in the sense that any equilibrium of our model can be translated into an equilibrium of the modified model and vice versa. In our model, the advertising strategy can be conditioned on the price using Bayes theorem as $\alpha(p)=\frac{\alpha f_{1}(p)}{\alpha f_{1}(p)+(1-\alpha) f_{0}(p)}$ and the two price distributions naturally can be combined as $F(p)=\alpha F_{1}(p)+(1-\alpha) F_{0}(p)$. In Robert and Stahl's way of modelling, the probability of advertising is $\alpha=\int \alpha(p) d F(p)$, the price distribution conditional on not advertising is $F_{0}(p)=\frac{\int_{0}^{p} 1-\alpha(x) d F(x)}{\int 1-\alpha(p) d F(p)}$ and the price distribution conditional on advertising is $F_{1}(p)=\frac{\int_{0}^{p} \alpha(x) d F(x)}{\int \alpha(p) d F(p)}$. Some of these results are also derived in Robert and Stahl's paper. More details on this conversion are available upon request.

${ }^{6}$ The support of a price distribution $F(p)$ are all prices $p$ where $\frac{\partial F(p)}{\partial p} \neq 0$.
} 
searched for. Based on this set, the consumer decides whether to search further or to stop searching. If he decides to stop searching, the product is bought from the firm with the lowest price lower than $\theta$ in the set of already obtained price quotations.

All players (firms and consumers) are rational and they all seek to maximize their profit or utility. The rationality of the players and the structure of the game is common knowledge. In this paper we will look for a symmetric perfect Bayesian equilibrium. In the remainder we will therefore drop the index $j$. The profit $\pi_{0}(p)$ denotes the expected profit when a firm does not advertise and charges price $p$ and $\pi_{1}(p)$ denotes the expected profit when advertising and charging price $p$. We define $\pi_{0}=\pi_{0}(p)$ for all $p$ in the support of $F_{0}(p)$ and $\pi_{1}=\pi_{1}(p)$ for all $p$ in the support of $F_{1}(p)$, so $\pi_{0}$ is the expected profit from not advertising and $\pi_{1}$ is the expected profit from advertising. Whenever $\alpha>0, \pi_{0}=\pi_{1}$.

\section{Equilibria}

We will first focus on the search behavior of the consumers. In the second part of this section firm behavior will be specified. Some observations on firm behavior will however be useful in the derivation of the consumer behavior.

Lemma 3.1 Firms will never set a price equal to 0 or above $\theta$.

The proof is available on request. The main idea incorporated in the proof is that profits are equal to 0 if a firm sets a price equal to 0 or above $\theta$. Setting a positive price below $\theta$ and not advertising yields strictly positive profits.

Since the price will never be above $\theta$, consumers will always buy when they have obtained one or more price quotations and have stopped searching.

\subsection{Consumer behavior}

For the non-shoppers we derive the optimal behavior conditional on a set of price quotations the consumer has already observed. Note that this set can be empty when no ads have been received and no firms have been searched. Assume for the moment that the set is non-empty. If the lowest price in the set is below a certain reservation price $r_{0}$ the consumer will not search further and buy at this lowest priced firm. If the lowest price in this set is however above $r_{0}$ and there are one or more firms still to be searched, the consumer will choose a firm from which it did not obtain a price quotation at random and will add the price found to the set of already obtained prices. If there are no more firms to be searched the consumer will buy at the lowest 
priced firm..$^{7,8}$

To specify the reservation price $r_{0}$ assume the lowest price in the set is given by $\hat{p}$. The expected gain from searching once more is given by

$$
\int_{\underline{p}_{0}}^{\hat{p}}(\hat{p}-p) d F_{0}(p)
$$

This expression shows that the expected gain arises when the price found is below $\hat{p}$. Note that $F_{0}(p)$ is used: only non-advertising firms are searched. The above expression can be integrated in parts and simplifies to

$$
\int_{\underline{p}_{0}}^{\hat{p}} F_{0}(p) d p .
$$

This defines $r_{0}$ as $\int_{p_{0}}^{r_{0}} F_{0}(p) d p=c$. One can easily see that for prices below $r_{0}$ it is not profitable to search: the expected gain is lower than the search costs. For prices above $r_{0}$ the expected gain exceeds the search costs and so searching is profitable. It follows that when search costs are low, the reservation price is low as well implying that consumers easily continue searching if prices are not low.

We assumed that the set with already obtained price quotations was nonempty. If this set is empty, consumers will search for sure if $r_{0}<\theta$. If $r_{0}=\theta$ they will search with probability $\mu \leq 1$, and if $r_{0}>\theta$ they will not search at all. To see this, note that the expected gain from searching when no price has yet been obtained is given by expression (1) where $\hat{p}$ is replaced by $\theta$. This implies that if and only if $\theta>r_{0}$ searching will be profitable. Hence, $\mu=1$ when $r_{0}<\theta, 0 \leq \mu \leq 1$ when $r_{0}=\theta$ and $\mu=0$ when $r_{0}>\theta$.

\subsection{Firm behavior}

In this section we will derive some general results on firm behavior that will be helpful in the next section where we derive the equilibria of our model.

Lemma 3.2 In a symmetric equilibrium, $\alpha<1$.

The main idea behind this lemma can be explained as follows. If both firms advertise for sure, all consumers will be aware of all prices in the market.

\footnotetext{
${ }^{7}$ Implicit in this specification is the assumption of a symmetric equilibrium. Symmetry implies that all non-advertising firms a priori are the same for the consumers. Therefore $r_{0}$ is not dependent on the firms that still have to be searched and consumers will pick a firm at random if they want to search.

${ }^{8}$ For completeness we assume that if the lowest price in the set equals $r_{0}$ consumers will not search on. We will see later however that the probability that the lowest price equals $r_{0}$ is 0 .
} 
Price will therefore be driven down to 0 (Bertrand outcome). The firms obtain negative payoffs $-A$ and so have an incentive not to advertise.

A corollary of lemmas 3.1 and 3.2 is that the expected profits of a nonadvertising firm charging $\underline{p}_{0}$ are strictly positive. It has positive probability that the competitor also does not advertise and so does not charge a price lower than $p_{0}$. This means that the non-advertising firm will with nonzero probability $1-\alpha$ attract at least half of the shoppers and so expects profit to be at least equal to $\underline{p}_{0}(1-\alpha) \frac{1}{2} \gamma>0$.

A second observation is that like many search papers, but unlike the paper by Robert and Stahl (1993), the price distributions are atomless. Also, prices that are chosen will never be larger than the reservation price of nonshoppers. These results are a consequence of the assumptions that there exists a mass of fully informed consumers and that consumers have to pay a search cost only when they have not yet observed a firm's price.

Lemma $3.3 F_{0}(p)$ and $F_{1}(p)$ are atomless and $F_{0}\left(r_{0}\right)=F_{1}\left(r_{0}\right)=1$.

Hence, if consumers get one or more ads, they will not search further. If they do not receive an ad, they will search at most once. The last result says that between the lower and upper bounds of the supports of the price distributions the profits are constant.

Lemma 3.4 For all prices between and including $\underline{p}_{0}$ and $\bar{p}_{0}, \pi_{0}(p)$ is constant and equal to $\pi_{0}$. For all prices between and including $\underline{p}_{1}$ and $\bar{p}_{1}, \pi_{1}(p)$ is constant and equal to $\pi_{1}$.

\subsection{Characterization of Equilibria}

The model has four exogenous parameters: $\theta, c, A$ and $\gamma$. It is possible to scale $\theta, c$ and $A$, that is, a model with these parameters multiplied by a constant gives the same equilibria, except that all prices (and $r_{0}$ ) and profits are multiplied by the same constant. For convenience, we will in the remainder of the paper scale all parameters with respect to $\theta$, and so we use $c$ and $A$ relative to $\theta$, and set $\theta=1$.

We first provide a classification of the different types of equilibria that may arise in our model and then characterize the equilibrium strategies of firms and consumers in each of these cases. The following theorem shows that three types of equilibria may arise in our model.

Theorem 3.5 Each symmetric equilibrium can be classified in one of three different types. Type I has firms not advertising at all $(\alpha=0)$. Type II has firms advertising with strictly positive probability $(0<\alpha<1)$ and 
overlapping supports of the price distributions with $\bar{p}_{0}=\bar{p}_{1}$. Type III has firms advertising with strictly positive probability $(0<\alpha<1)$ and price distributions that do not overlap. In particular, $\bar{p}_{0}=\underline{p}_{1}$ and so advertised prices are higher than non-advertised prices.

Note that in type III equilibria, advertised prices are always higher than non-advertised prices. The reverse, advertised prices always being below non-advertised prices, cannot arise in equilibrium. In the Appendix we show that if advertised prices are always lower than non-advertised prices, an advertising firm has an incentive to deviate and advertise the highest nonadvertised price. This leads to somewhat less sales, but at a much higher price.

Each of the three types of equilibria has a corresponding parameter region where the equilibrium exists. These regions will be expressed in terms of $A$ and $c$, taking $\gamma$ constant. The derivation of the equilibria and corresponding parameter regions will show that each parameter set $[A, c, \gamma]$ has a unique corresponding symmetric equilibrium.

Each symmetric equilibrium of a certain type can be further classified in one of two cases. Case $a$ has $0<\mu<1$ and case $b$ has $\mu$ equal to 1 (for type $I$ and $I I$ ) or 0 (for type $I I I$ ). To see that in equilibrium type $I$ an equilibrium with $\mu=0$ does not exist, note that a type $I$ equilibrium has $\alpha=0$. No search would imply firms only sell to shoppers, and so prices would be 0 . This contradicts the no-search assumption. In equilibrium type $I I$ an equilibrium with $\mu=0$ also does not exist. If there were such an equilibrium, a non-advertising firm charging $\bar{p}_{0}$ would obtain zero profit, contradicting the discussion immediately after lemma 3.2. In equilibrium type $I I I$ an equilibrium with $\mu=1$ does not exist. If there were such an equilibrium, the requirement $\pi_{1}\left(r_{0}\right)=\pi_{1}\left(p_{1}\right)$ would give rise to the condition $r_{0}(1-\alpha)(1-$ $\gamma)-A=\underline{p}_{1}(\alpha+(1-\alpha)(1-\gamma))-A$. On the other hand, it is easy to see that in such a situation $\pi_{0}\left(r_{0}\right)=\frac{1}{2} r_{0}(1-\alpha)(1-\gamma)$, which given this condition is larger than $\underline{p}_{1}\left(\gamma \alpha+\frac{1}{2}(1-\alpha)(1-\gamma)\right)=\pi_{0}\left(\underline{p}_{1}\right)$. Hence, the non-advertising firm would have an incentive to deviate and charge the reservation price. Therefore, we conclude that each of the three types of equilibrium classified in the theorem, can be further divided into two subcategories, depending on whether $\mu=0,0<\mu<1$, or $\mu=1$.

For each of the three types of equilibrium, we will now derive case $a$. The derivation of case $b$ is very similar in nature and can be found in the Appendix.

Equilibrium type I: no advertising $(\alpha=0) .{ }^{9}$

As indicated above when characterizing optimal search behavior, $0<\mu<1$

\footnotetext{
${ }^{9}$ Note that the assumption $\alpha=0$ gives exactly the same model as in Janssen, MoragaGonzalez and Wildenbeest (2004).
} 
implies $r_{0}=1 .{ }^{10}$ Furthermore, in this case of no advertising, a firm's profit equals

$$
\pi_{0}(p)=p\left[(1-\gamma) \frac{1}{2} \mu+\gamma\left(1-F_{0}(p)\right)\right] .
$$

This expression consists of two parts. The non-shoppers search at random and do not search further; this gives each firm a fraction $\frac{1}{2}$ of the nonshoppers. The shoppers buy at the lowest-priced firm; they are attracted if and only if the competing firm charges a higher price, which happens with probability $1-F_{0}(p)$. A maximum price below $r_{0}$ can not be part of an equilibrium since deviating to a price of $r_{0}$ would then be profitable. So, $\bar{p}_{0}=$ $r_{0}$. Charging the maximum price gives expected profit $\pi_{0}\left(r_{0}\right)=r_{0} \frac{1}{2} \mu(1-\gamma)$. Equating $\pi_{0}(p)$ and $\pi_{0}\left(r_{0}\right)$ gives the equilibrium price distribution:

$$
F_{0}(p)=1-\frac{\left(r_{0}-p\right) \frac{1}{2} \mu(1-\gamma)}{\gamma p}
$$

which has as its lower bound $\underline{p}_{0}=\frac{r_{0} \mu(1-\gamma)}{2 \gamma+\mu(1-\gamma)}$. Note that this price distribution is strictly increasing for $p \in\left[\underline{p}_{0}, r_{0}\right]$ and so does not have a 'gap'. It is clear that deviating to lower prices is not profitable.

Since $r_{0}=1, \int_{\underline{p}_{0}}^{r_{0}} F_{0}(p) d p=c$ is an expression in $\gamma, c$ and $\mu$. This expression provides an implicit definition of $\mu$ :

$$
\mu \ln \frac{\mu(1-\gamma)}{2 \gamma+\mu(1-\gamma)}=(c-1) \frac{2 \gamma}{1-\gamma} .
$$

Note that $\mu \ln \frac{\mu(1-\gamma)}{2 \gamma+\mu(1-\gamma)}$ is strictly decreasing from 0 to $\ln \frac{1-\gamma}{1+\gamma}$ for $0 \leq \mu \leq 1$ so that $\mu$ is uniquely defined.

For $\alpha=0$ to hold, advertising should not be profitable. The expected profit from advertising a price $p$ is given by $p\left[(1-\gamma)+\gamma\left(1-F_{0}(p)\right)\right]-A=(1-\gamma)(p+$ $\left.\frac{1}{2} \mu r_{0}-\frac{1}{2} p \mu\right)-A$. This expected profit is maximized for $p=r_{0}$, giving profit $r_{0}(1-\gamma)-A$. Advertising is not profitable when $r_{0}(1-\gamma)-A<r_{0}(1-\gamma) \frac{1}{2} \mu$. Rearranging terms and using $r_{0}=1$ gives

$$
\mu>2\left(1-\frac{A}{1-\gamma}\right)
$$

The two assumptions $0<\mu<1$ and $\alpha=0$ together hold when

$$
\max \left\{0,2\left(1-\frac{A}{1-\gamma}\right)\right\}<\mu<1 .
$$

\footnotetext{
${ }^{10}$ To simplify the derivation of equilibrium $I b$, we will not yet plug $r_{0}=1$ in the equilibrium expressions for equilibrium $I a$. The same holds for equilibrium IIa.
} 
Using the definition of $\mu$ given by (2) and the fact that $\mu \ln \frac{\mu(1-\gamma)}{2 \gamma+\mu(1-\gamma)}$ is strictly decreasing for $0<\mu<1$, this gives rise to the following parameter restrictions:

$$
\beta_{1} \equiv 1+\frac{1-\gamma}{2 \gamma} \ln \frac{1-\gamma}{1+\gamma}<c<\min \left\{1,1+\frac{1-\gamma-A}{\gamma} \ln \frac{1-\gamma-A}{1-A}\right\} .
$$

As $0<\beta_{1}<1$, it is clear that this type of equilibrium exists whenever $\frac{1-\gamma}{2}<A$.

The above discussion, and the derivation of equilibrium $I b$ in the Appendix can be summarized as follows.

Proposition 3.6 An equilibrium with $\alpha=0$ has

$$
F_{0}(p)=1-\frac{\left(r_{0}-p\right) \frac{1}{2} \mu(1-\gamma)}{\gamma p} .
$$

If $\beta_{1}<c<\min \left\{1,1+\frac{1-\gamma-A}{\gamma} \ln \frac{1-\gamma-A}{1-A}\right\}$ (where $\beta_{1}$ is defined in (3)), $r_{0}=1$ and $\mu$ is implicitly defined by

$$
\mu \ln \frac{\mu(1-\gamma)}{2 \gamma+\mu(1-\gamma)}=(c-1) \frac{2 \gamma}{1-\gamma} .
$$

If $c<\min \left\{\beta_{1}, \frac{2 A \beta_{1}}{1-\gamma}\right\}, \mu=1$ and $r_{0}=\frac{c}{\beta_{1}}$.

Equilibrium type II: some advertising $(0<\alpha<1)$ and partially overlapping price distributions $\left(\bar{p}_{0}=\bar{p}_{1}\right)$.

First note that since $0<\mu<1$ the reservation price for non-shoppers should be equal to the consumers' valuation in this case, i.e., $r_{0}=1$. Furthermore, the profit equations in the case of non-advertising and advertising are equal to

$$
\pi_{0}(p)=p\left[\gamma \alpha\left(1-F_{1}(p)\right)+\gamma(1-\alpha)\left(1-F_{0}(p)\right)+(1-\gamma)(1-\alpha) \frac{1}{2} \mu\right],
$$

respectively,

$$
\pi_{1}(p)=p\left[\alpha\left(1-F_{1}(p)\right)+\gamma(1-\alpha)\left(1-F_{0}(p)\right)+(1-\gamma)(1-\alpha)\right]-A .
$$

These equations have a similar interpretation as above. A firm only attracts all shoppers if it has the lowest price taking into account that the competitor may charge different prices depending on whether or not it advertises. In case the firm does not advertise, it attracts only half of the non-shoppers that do search themselves and only when the competitor does not advertise. 
The advertising firm attracts more consumers, namely all non-shoppers if the competitor does not advertise or if the competitor advertises a higher price, and has to pay the advertising $\operatorname{cost} A$.

Whenever the upper bounds of the two price distributions are equal we can use standard arguments to show that the upper bound then has to be equal to the reservation price, i.e., $\bar{p}_{0}=\bar{p}_{1}=r_{0}$. As this upper bound is in both price distributions, $\pi_{0}\left(r_{0}\right)$ has to be equal to $\pi_{1}\left(r_{0}\right)$, which gives the condition

$$
r_{0}(1-\gamma)(1-\alpha)\left(1-\frac{1}{2} \mu\right)=A
$$

Using lemma 3.4 note that $\pi_{0}(p)=\pi_{0}$ and $\pi_{1}(p)=\pi_{1}$ for all prices higher than $\max \left\{\underline{p}_{0}, \underline{p}_{1}\right\}$. In this price region we therefore can use $\pi_{0}(p)=\pi_{1}(p)$ to derive

$$
F_{1}(p)=1-\frac{A-\left(1-\frac{1}{2} \mu\right) p(1-\alpha)(1-\gamma)}{\alpha(1-\gamma) p}=1-\frac{\left(r_{0}-p\right)(1-\alpha)\left(1-\frac{1}{2} \mu\right)}{\alpha p}
$$

Using $\pi_{0}(p)=\pi_{0}\left(r_{0}\right)$ and the above expression for $F_{1}(p)$ we can also derive that

$F_{0}(p)=1-\frac{r_{0}(1-\gamma)(1-\alpha) \frac{1}{2} \mu-\frac{A \gamma}{1-\gamma}+p(1-\alpha)\left(\gamma-\frac{1}{2} \mu\right)}{\gamma(1-\alpha) p}=1-\frac{\left(r_{0}-p\right)\left(\frac{1}{2} \mu-\gamma\right)}{\gamma p}$.

We stress that these price distributions only hold for prices that are equal to or larger than $\max \left\{\underline{p}_{0}, \underline{p}_{1}\right\}$. Note that the price distributions do not have a 'gap', that is, they are strictly increasing for $p \in\left[\max \left\{\underline{p}_{0}, \underline{p}_{1}\right\}, r_{0}\right]$, so the supports of $F_{0}(p)$ and $F_{1}(p)$ overlap in this price region. We can now distinguish two cases: $(i), \underline{p}_{0}<\underline{p}_{1}$ and $(i i), \underline{p}_{0}>\underline{p}_{1}$.

We first look at case $(i)$. In this case, $F_{1}(p)$ is as defined above, and $F_{0}(p)$ is as defined above for $p>\underline{p}_{1}=\frac{A}{(1-\gamma)\left(1-\frac{1}{2} \mu(1-\alpha)\right)}$, where this expression can be obtained by setting $F_{1}(p)=0$ in $(5)$. For $p<\underline{p}_{1}$, we can use $\pi_{0}(p)=\pi_{0}\left(r_{0}\right)$ to get the price distribution

$$
F_{0}(p)=1-\frac{\left(r_{0}-p\right)(1-\gamma)(1-\alpha) \frac{1}{2} \mu-p \gamma \alpha}{p \gamma(1-\alpha)},
$$

with $\underline{p}_{0}=\frac{r_{0} \frac{1}{2} \mu A}{\frac{1}{2} \mu A+r_{0}\left(1-\frac{1}{2} \mu\right) \gamma}$.

Case $(i i)$ can be derived in the same way as case $(i)$. We then get that

$$
F_{1}(p)=1-\frac{r_{0}(1-\gamma)(1-\alpha)-p(1-\alpha)}{\alpha p}
$$


for $p<\underline{p}_{0}$ and $\underline{p}_{1}=r_{0}(1-\gamma)(1-\alpha)$.

To check under which conditions an equilibrium of type II $I$ exists, we first note that it is not profitable to charge a price below $\underline{p}_{0}$ without advertising and that it also is not profitable to advertise a price below $\underline{p}_{1}$. In addition, the following parameter restrictions have to hold. First, $\mu$ should be between 0 and 1. Furthermore, $\alpha$ as defined by (4) should also be between 0 and 1 . This gives rise to condition $\mu<2-\frac{2 A}{1-\gamma}$. Finally, it should be that $\mu>2 \gamma$, since otherwise $F_{0}(p)$ is decreasing in $p$. Thus, we have that $\mu$ should satisfy $2 \gamma<\mu<\min \{1,2-2 A /(1-\gamma)\}$.

For equilibrium IIai, $\int_{\underline{p}_{0}}^{r_{0}} F_{0}(p) d p=c$ and $r_{0}=1$ gives

$$
1+\frac{1-\gamma}{2 \gamma} \mu \ln \frac{\frac{1}{2} \mu A}{\frac{1}{2} \mu A+\left(1-\frac{1}{2} \mu\right) \gamma}-\left(1-\frac{1}{2} \mu\right) \ln \frac{A\left(1-\frac{1}{2} \mu\right)}{(1-\gamma)\left(1-\frac{1}{2} \mu\right)-A \frac{1}{2} \mu}=c
$$

which implicitly defines $\mu$ as a function of $A, c$ and $\gamma$. For equilibrium IIaii, $\int_{\underline{p}_{0}}^{r_{0}} F_{0}(p) d p=c$ and $r_{0}=1$ gives

$$
1-\frac{\gamma-\frac{1}{2} \mu}{\gamma} \ln \frac{\mu-2 \gamma}{\mu}=c
$$

again implicitly defining $\mu$ as function of $c$ and $\gamma$. The L.H.S. of expressions (9) and (10) are both decreasing in $\mu$ and so $2 \gamma<\mu<\min \{1,2-2 A /(1-\gamma)\}$ can be rewritten as

$$
\begin{aligned}
\max \left\{1+\frac{1-\gamma}{2 \gamma} \ln \frac{A}{A+\gamma}-\frac{1}{2} \ln \right. & \left.\frac{A}{1-\gamma-A}, 1+\frac{1-\gamma-A}{\gamma} \ln \frac{1-\gamma-A}{1-A}\right\} \\
& <c<1+(1-\gamma) \ln \frac{(1-\gamma)^{2}-A \gamma}{A(1-\gamma)+(1-\gamma)^{2}}
\end{aligned}
$$

for case $(i)$, and

$$
\max \left\{\beta_{2}, 1+\frac{(1-\gamma)^{2}-A}{\gamma(1-\gamma)} \ln \frac{(1-\gamma)^{2}-A}{1-\gamma-A}\right\}<c<1
$$

for case $(i i)$, where $\beta_{2} \equiv 1+\frac{1-2 \gamma}{2 \gamma} \ln (1-2 \gamma)$. For equilibrium type $I I a$ to hold either restriction (11) or restriction (12) should hold. However, note that for restriction (11) to be relevant, $\underline{p}_{0}$ should be smaller than $\underline{p}_{1}$, while for restriction (12), $\underline{p}_{0}$ should be larger than $\underline{p}_{1}$. In the Appendix we show that the resulting conditions can be simplified to the ones mentioned in Proposition 3.7.

Proposition 3.7 An equilibrium with $0<\alpha<1$ and $\bar{p}_{0}=\bar{p}_{1}$ has $\alpha=$ $1-\frac{A}{r_{0}(1-\gamma)\left(1-\frac{1}{2} \mu\right)}$ and $F_{0}(p)$ and $F_{1}(p)$ being defined by (6) and (5) respectively 
in the common support $\left[\max \left\{\underline{p}_{0}, \underline{p}_{1}\right\}, r_{0}\right]$ and (7) and (8) respectively for $p<\underline{p}_{1}$ and $p<\underline{p}_{0}$.

(i) If either

$$
A<\frac{1-2 \gamma}{2} \text { and } \beta_{2}<c<1+(1-\gamma) \ln \frac{(1-\gamma)^{2}-A \gamma}{A(1-\gamma)+(1-\gamma)^{2}}
$$

or, $A>\frac{1-2 \gamma}{2}$ and

$\max \left\{1+\frac{1-\gamma}{2 \gamma} \ln \frac{A}{A+\gamma}-\frac{1}{2} \ln \frac{A}{1-\gamma-A}, 1+\frac{1-\gamma-A}{\gamma} \ln \frac{1-\gamma-A}{1-A}\right\}<c<$

$$
1+(1-\gamma) \ln \frac{(1-\gamma)^{2}-A \gamma}{A(1-\gamma)+(1-\gamma)^{2}}
$$

$0<\mu<1$ in which case $r_{0}=1$ and $\mu$ is defined by equations (9) for the case where $\underline{p}_{0}<\underline{p}_{1}$ and by (10) for the case where $\underline{p}_{1}<\underline{p}_{0}$.

(ii) If $A<\frac{1-2 \gamma}{2}$ and $\frac{2 A \beta_{1}}{1-\gamma}<c<\beta_{2}$ or $A>\frac{1-2 \gamma}{2}$ and

$$
\frac{2 A \beta_{1}}{1-\gamma}<c<1+\frac{1-\gamma}{2 \gamma} \ln \frac{A}{A+\gamma}-\frac{1}{2} \ln \frac{A}{1-\gamma-A},
$$

$\mu=1$ and $r_{0}$ is implicitly defined by

$$
\frac{r_{0}}{2} \ln \left(\frac{r_{0}}{A}(1-\gamma)-1\right)-\frac{r_{0}(1-\gamma)}{2 \gamma} \ln \left(\frac{r_{0}}{A} \gamma+1\right)+r_{0}=c
$$

for the case where $\underline{p}_{0}<\underline{p}_{1}$ and by $r_{0}=\frac{c}{\beta_{2}}$ for the case where $\underline{p}_{0}>\underline{p}_{1}$.

Equilibrium type III : some advertising $(0<\alpha<1)$ and price distributions that do not overlap $\left(\bar{p}_{0}=\underline{p}_{1}\right)$.

Finally, we will turn to the last type of equilibrium, namely the one where firms spend some money on advertising and when they do advertise, they set consistently higher prices than when they don't advertise, i.e., $\bar{p}_{0}=\underline{p}_{1}$. Using standard arguments, we first observe that $\bar{p}_{1}=r_{0}=1$. Profits in case of advertising are now given by the following expression

$$
\pi_{1}(p)=p \alpha\left(1-F_{1}(p)\right)+p(1-\alpha)(1-\gamma)-A .
$$

This expression can be understood as follows. When a firm advertises, it knows it gets all consumers when its competitor also advertises and sets a higher price. When the competitor does not advertise, he always asks a lower price and so shoppers will buy from him. Non-shoppers however do not search after receiving an ad and buy from the advertising firm. In order to derive the equilibrium price distribution in case of advertising, we equate this expression with the profits the firm gets when advertising the reservation price: $\pi_{1}(1)=(1-\alpha)(1-\gamma)-A$. This yields the following expression 


$$
F_{1}(p)=1-\frac{(1-p)(1-\alpha)(1-\gamma)}{p \alpha}
$$

with $\underline{p}_{1}=\frac{(1-\alpha)(1-\gamma)}{\alpha+(1-\alpha)(1-\gamma)}$. Now note that the profits a firm gets from not advertising are given by

$$
\pi_{0}(p)=p \gamma \alpha+p \gamma(1-\alpha)\left(1-F_{0}(p)\right)+p(1-\gamma)(1-\alpha) \frac{1}{2} \mu .
$$

A non-advertising firm in this case gets shoppers if, and only if, the other firm advertises or the other firm does not advertise and sets a higher price. Non-shoppers will come to the shop only when the competitor also does not advertise and in that case, both firms receive half of the non-shoppers. It is easy to see that setting the highest non-advertised price yields a profit equal to $\pi_{0}\left(\bar{p}_{0}\right)=\bar{p}_{0} \gamma \alpha+\bar{p}_{0}(1-\gamma)(1-\alpha) \frac{1}{2} \mu$. Equating $\pi_{0}(p)$ and $\pi_{0}\left(\bar{p}_{0}\right)$ gives

$$
F_{0}(p)=1-\frac{\left(\bar{p}_{0}-p\right)\left(\gamma \alpha+(1-\gamma)(1-\alpha) \frac{1}{2} \mu\right)}{p \gamma(1-\alpha)} .
$$

It is easy to see that charging a price below $\underline{p}_{0}$ is never profitable. Advertising a price below $\underline{p}_{1}$ is also never profitable. ${ }^{11}$ It also should not be profitable to refrain from advertising and charge a price above $\bar{p}_{0}$. Note that for $p \geq \bar{p}_{0}$, $\pi_{0}(p)=p\left(\gamma \alpha\left(1-F_{1}(p)\right)+(1-\alpha)(1-\gamma) \frac{1}{2} \mu\right)$. Substituting the expression for $F_{1}(p)$ given in (13) yields an expression that is decreasing in $p$ whenever $\mu<2 \gamma$. Hence, this is a necessary condition for this equilibrium to exist.

For the above equilibrium to hold, there are some other parameter restrictions as well. First, $0<\alpha<1$ requires $\pi_{0}=\pi_{1}$, which gives

$$
\alpha^{2} \frac{1}{2} \mu(1-\gamma)+\alpha\left((1-\gamma)(1-\mu)+\frac{\gamma}{1-\gamma} A\right)+A-(1-\gamma)\left(1-\frac{1}{2} \mu\right)=0
$$

Furthermore, $0<\mu<1$ gives $\int_{\underline{p}_{0}}^{1} F_{0}(p) d p=c$. Substituting $F_{0}(p)$ gives

$$
1+\frac{(1-\alpha)(1-\gamma)-A}{\gamma(1-\alpha)} \ln \frac{\gamma \alpha+(1-\alpha)(1-\gamma) \frac{1}{2} \mu}{\gamma+(1-\alpha)(1-\gamma) \frac{1}{2} \mu}=c .
$$

Equations (15) and (16) together define $\alpha$ and $\mu$ as equations of $c, A$ and $\gamma$. For the equilibrium to hold, $0<\alpha<1$ and $0<\mu<2 \gamma$.

Note that equation (15) is an increasing function of $\alpha$, that reaches $A-(1-$ $\gamma)\left(1-\frac{1}{2} \mu\right)$ for $\alpha=0$ and $\frac{A}{1-\gamma}$ for $\alpha=1$. The restriction $0<\alpha<1$ therefore reduces to $A-(1-\gamma)\left(1-\frac{1}{2} \mu\right)<0$, which gives $\mu<2-\frac{2 A}{1-\gamma}$.

It can be shown that expression (16) is decreasing in $\mu$ and so the restrictions $0<\mu<\min \left\{2 \gamma, 2-\frac{2 A}{1-\gamma}\right\}$ can be written as

\footnotetext{
${ }^{11}$ To see this, write down $\pi_{1}(p)$ for $p \leq \underline{p}_{1}$ using the above expression for $F_{0}(p)$ and note that $\pi_{1}(p)$ is increasing in $p$.
} 


$$
\begin{array}{r}
\max \left\{1+(1-\gamma) \ln \frac{(1-\gamma)^{2}-A \gamma}{(1-\gamma)^{2}+A(1-\gamma)}, 1+\frac{(1-\gamma)-A}{\gamma} \ln \frac{(1-\gamma)-A}{1-A}\right\}<c< \\
1+((1-\gamma)-A) \ln \frac{(1-\gamma)^{2}-A(1-\gamma)}{(1-\gamma)^{2}+A \gamma}
\end{array}
$$

We summarize as follows:

Proposition 3.8 Equilibria with $0<\alpha<1$ and $\bar{p}_{0}=\underline{p}_{1}$ have $F_{1}(p)$ as defined in (13) and $F_{0}(p)$ as defined in (14) with $\bar{p}_{0}=\frac{(1-\alpha)(1-\gamma)}{\alpha+(1-\alpha)(1-\gamma)}$. If

$$
\begin{aligned}
& \max \left\{1+(1-\gamma) \ln \frac{(1-\gamma)^{2}-A \gamma}{(1-\gamma)^{2}+A(1-\gamma)}, 1+\right.\left.\frac{(1-\gamma)-A}{\gamma} \ln \frac{(1-\gamma)-A}{1-A}\right\}<c< \\
& 1+((1-\gamma)-A) \ln \frac{(1-\gamma)^{2}-A(1-\gamma)}{(1-\gamma)^{2}+A \gamma}
\end{aligned}
$$

we have $0<\mu<1$ and $\alpha$ and $\mu$ are implicitly defined by (15) and (16). If

$$
c>1+((1-\gamma)-A) \ln \frac{(1-\gamma)^{2}-(1-\gamma) A}{(1-\gamma)^{2}+\gamma A},
$$

$\mu=0$ and $\alpha$ is given by $\frac{(1-\gamma)^{2}-A(1-\gamma)}{(1-\gamma)^{2}+A \gamma}$.

In table 4 in the Appendix the equilibria are summarized.

\subsection{Discussion}

We will now first take a closer look at the parameter regions in which the different types of equilibria exist. Figures 1 and 2 depict for each equilibrium the region where it exists in terms of $A$ and $c$. We note that the regions do not overlap and that they together fill the complete parameter space. Figure 1 is drawn for $\gamma=0.1$, while figure 2 assumes $\gamma=0.4$. Equilibria IIa and $I I b$ are both divided by a dotted line. Left of this line, $\underline{p}_{1}<\underline{p}_{0}$, while right of this line $\underline{p}_{0}<\underline{p}_{1}$.

We note that for the equilibria with no advertising (equilibrium type $I$ ) to exist, the search costs $c$ should be low or the advertising costs $A$ should be high. The intuition is fairly simple: for high $A$ advertising is too expensive to be profitable. For low search cost, firms can not ask high prices since otherwise the consumers will search on and so advertising is too expensive relative to the prices that can be asked. 


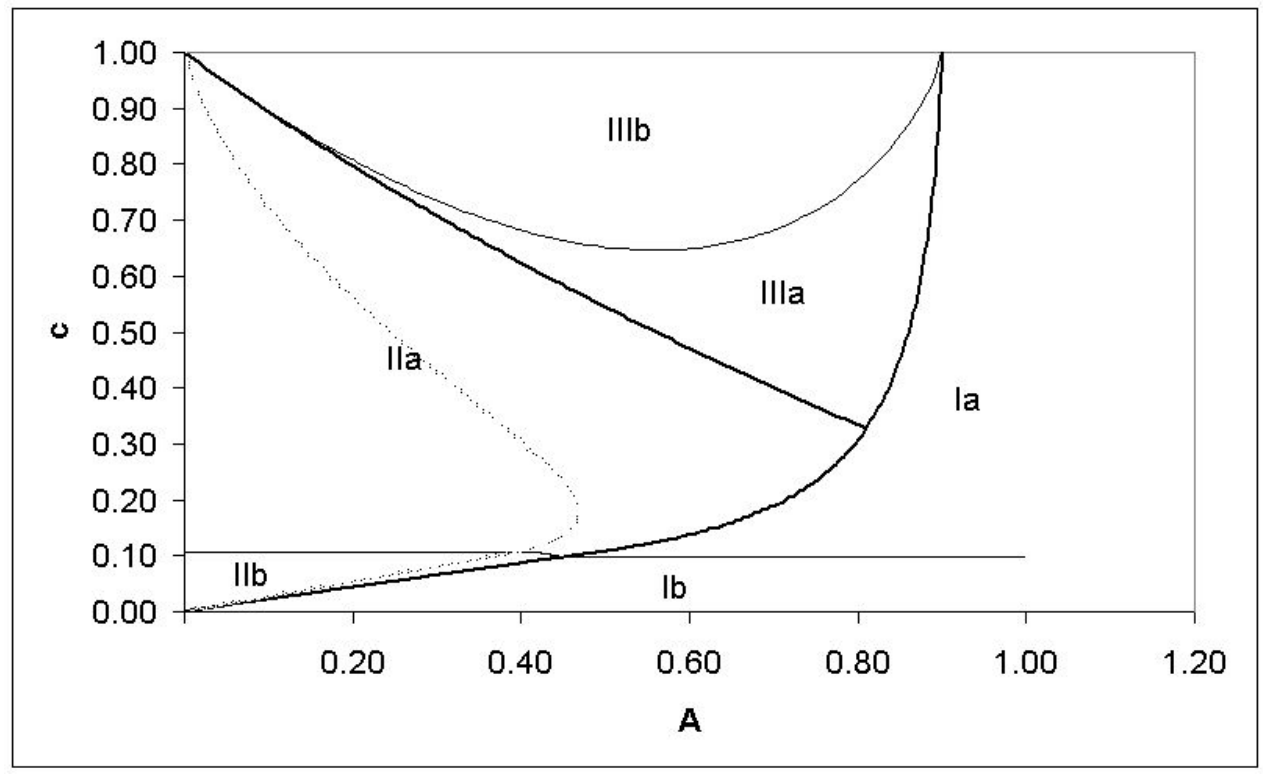

Figure 1: The parameter regions in terms of $A$ and $c$ where the different equilibria exist, assuming $\gamma=0.1$. Each region is numbered according to the type of equilibrium that exists in that region.

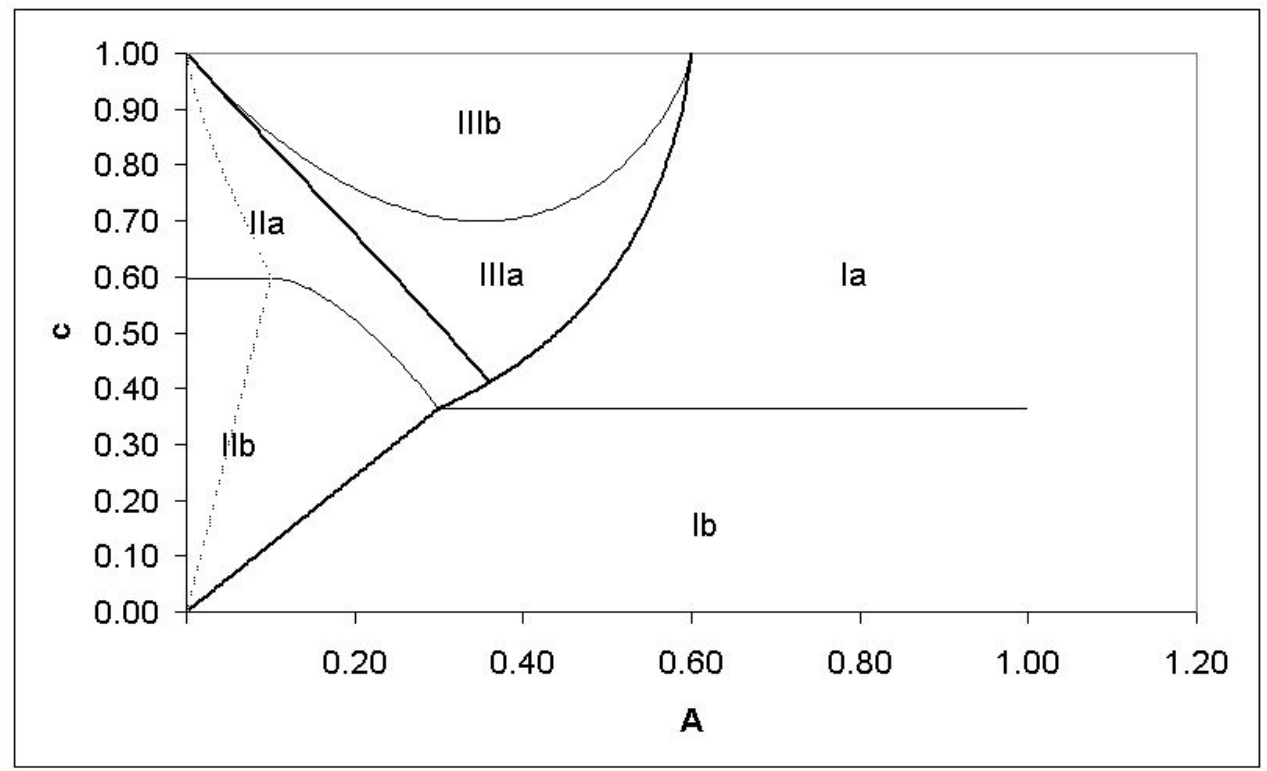

Figure 2: The parameter regions in term of $A$ and $c$ where the different equilibria exist, assuming $\gamma=0.4$. Each region is numbered according to the type of the equilibrium that exists in that region. 
The equilibria with full consumer search (equilibrium types $I b$ and $I I b$ where $\mu=1)$ are in a region with low search costs, whereas their counterparts with partial consumer search $(0<\mu<1)$ are in a region with higher search cost. The equilibrium with no consumer search (equilibrium type $I I I b$ ) only exists for $c$ relatively high and $A$ not too low or high. It is clear that higher search cost lead to less search. The intuition for the restriction on $A$ is as follows. For high $A$ it is not profitable to advertise and so firms only sell to the shoppers and searching consumers. Note that if consumers do not search at all, prices and profits will be driven to 0 , giving consumers an incentive to search. For low $A$ firms have a large incentive to advertise, which drives prices down. This leads to a higher payoff from search and therefore to some consumer search even if the search costs are large.

We note that if firms do not advertise $(\alpha=0)$, the non-shoppers search with strictly positive probability as long as the search cost $c$ are below the valuation for the product (see also Janssen, Moraga and Wildenbeest (2004)). On the other hand, if the non-shoppers do not search $(\mu=0)$, the firms advertise with strictly positive probability as long as the advertising costs are below $1-\gamma$. This is in contrast with the Robert and Stahl model, where no advertising implies no consumer search and a strictly positive probability of advertising leads to a strictly positive probability that consumers search. These differences are driven by the assumption of a non-zero segment of shoppers and the assumption that consumers only have to pay search costs when searching a shop and not when visiting an advertising firm. First, if there are no shoppers, no advertising and a positive probability of consumer search gives firms monopoly power over the searching consumers, leading to prices equal to $\theta$, contradicting the assumption of consumer search. If on the other hand there are shoppers, the firms have an incentive to compete, leading to lower prices and thus facilitating consumer search. Second, if consumers have to pay search costs for every first visit to a shop, firms do advertise with strictly positive probability and consumers do not search, firms will never ask a price above $1-c,{ }^{12}$ contradicting the assumption of no search. If consumers on the other hand only have to pay the search costs when searching a shop, firms will never ask a price above 1 , which is not contradicting the no-search assumption.

We will now discuss the equilibrium price distributions that arise. Note that when the search costs $c$ are low or high or the advertising costs $A$ are high, $\underline{p}_{0}<\underline{p}_{1}$. For intermediate $c$, and low $A, \underline{p}_{0}>\underline{p}_{1}$. This can be explained by the tradeoff firms have to make. Advertising firms attract the

\footnotetext{
${ }^{12}$ Advertising a higher price leads to no sales and a negative profit, asking a higher price without advertising leads to no sales and a zero profit. However, the profit of asking a price below $1-c$ is strictly positive.
} 
non-shoppers for sure if the competitor does not advertise, but they always have to compete for the shoppers and have to compete for the non-shoppers if the competitor advertises. Non-advertising firms have to compete for the shoppers, but attract half of the searching non-shoppers if the competitor also does not advertise. When $c$ is low or $A$ is high, $\alpha$ is low. This means that an advertising firm with large probability attracts the non-shoppers and only has to compete for the shoppers. A non-advertising firm also has to compete for the shoppers, but attracts only half of the searching non-shoppers. Therefore, a non-advertising firm has a greater incentive to compete and to lower its price. On the other hand, when $c$ is high, the probability with which non-shoppers search, $\mu$, is low and so non-advertising firms mainly sell to the shoppers, for which they have to compete. This leads to fierce competition, and low prices conditional on not advertising. These observations on the equilibrium price distributions differ from the Robert and Stahl (1993) result as explained in the Introduction.

\section{Comparative Statics}

In this section we will give some asymptotic and comparative static results. We are interested in the impact of changes in the three parameters $c, A$ and $\gamma$ of the model on the variables that are of main interest such as prices, profits and welfare. We also provide a discussion on the impact on the intensity with which consumers search or the intensity with which firms advertise as these variables are important in understanding the results. We start the discussion with some limiting results.

Theorem 4.1 a When c becomes arbitrarily small, firms do not advertise and there is full consumer search $(\mu=1)$. The maximum price charged approaches 0 .

b When $A$ becomes arbitrarily small, the advertising intensity $\alpha$ converges to 1 and the expected advertised price E $E p_{1}$ converges to 0 .

c When $\gamma$ becomes arbitrarily small, the advertising intensity $\alpha$ converges to $1-A$ and $\mu$ becomes arbitrarily close to 0 . Furthermore, $F_{1}(p) \rightarrow$ $1-\frac{A(1-p)}{p(1-A)}$.

These results can be understood as follows. When the search costs approach 0, the Bertrand result arises. This asymptotic result also occurs in the pure search models of Stahl (1989) and Janssen, Moraga-Gonzalez and Wildenbeest (2004). The intuition is simple: if the search costs are very low, consumers are almost always willing to search and to prevent further search, firms lower their prices, in that way preventing advertising. This Bertrand result however does not arise in Robert and Stahl's model. In their model, 
when the search costs approach zero, advertising diminishes, but the minimum price is strictly above zero. This difference in results is caused by the fact that there is a shopper segment in our model and not in Robert and Stahl (1993). Without shoppers, Robert and Stahl obtain a Diamond type of result, namely that when $c$ vanishes, the equilibrium price distribution converges to a degenerate distribution where all firms charge one particular, strictly positive, price for sure. Given that all firms charge the same price, no consumer wants to continue searching even at arbitrarily small search costs. A fraction of shoppers, however small, causes a breakdown of this result.

When the cost of advertising $A$ approaches 0 , we reach the same asymptotic results as in Stahl's pure advertising model (1994) and Robert and Stahl's advertising and search model: all firms advertise almost for sure, and prices become small. The intuition is simple. When advertising is virtually costless, every firm is willing to advertise, leading to fierce competition and decreasing prices.

When the shopper segment vanishes, the results are clearly different from the results of Stahl's pure search model with vanishing shopper segment, where the Diamond (monopoly pricing) result arises. The difference between our model and the pure search models is formed by the possibility to advertise. Our result replicates the result in Stahl's pure advertising model, which assumes no shopper segment. Robert and Stahl yield again a different conclusion mainly due to differences in the advertising cost function. ${ }^{13}$

We next proceed to a full comparative statics analysis. The analysis provides an assessment of the impact of a change in exogenous parameter values on the economic variables of interest $\alpha, \mu$, the expected non-advertised price $E p_{0}$, the expected advertised price $E p_{1}$, the profit $\pi$ and welfare conditional on a certain type of equilibrium to be present in the economy. Note that in the equilibria with partial consumer search $(0<\mu<1), \theta-E p_{0}-c=0$. Furthermore, in equilibria $I$ and $I I$ the profit equals $r_{0} \frac{1}{2} \mu(1-\alpha)(1-\gamma)$, while in equilibrium $I I I$ profit equals $r_{0}(1-\alpha)(1-\gamma)-A$. We define expected welfare as the value of all transactions taking place minus the search and advertising costs that have been incurred, i.e, expected welfare equals

$$
1-(1-\gamma)(1-\mu)(1-\alpha)^{2}-2 \alpha A-(1-\gamma) \mu(1-\alpha)^{2} c .
$$

\footnotetext{
${ }^{13}$ Note that with linear advertising costs the absence of shoppers implies that the price distribution conditional on not advertising is degenerate in the reservation price. With $\gamma=0$ and linear advertising costs therefore there is no consumer search. In the Robert and Stahl model with $\gamma=0$ there is consumer search because the convex advertising cost function prevents a firm advertising a low price from advertising to the complete market. Consumers that do not receive an ad therefore have a positive probability of finding a low price and have an incentive to search.
} 


\begin{tabular}{|c|cccccc|}
\hline & $I a$ & $I b$ & $I I a$ & $I I b$ & $I I I a$ & $I I I b$ \\
\hline$\alpha$ & 0 & 0 & + & + & + & 0 \\
$\mu$ & - & 0 & - & 0 & - & 0 \\
$E p_{0}$ & - & + & - & $+/-$ & - & 0 \\
$E p_{1}$ & & & $-*$ & + & - & 0 \\
$\pi$ & - & + & - & 0 & - & 0 \\
Welfare & - & - & - & - & $-*$ & 0 \\
\hline
\end{tabular}

Table 1: Local comparative statics results with respect to changes in $c$. A* denotes that numerical methods have been used to obtain the result.

In figures 3 and 4 we give a description of how for example, expected price depends on search cost across equilibria for specific values of the other exogenous parameters. Tables 1 to 3 provide an overview of the local comparative statics. $\mathrm{A}^{\prime}+{ }^{\prime}$ in these tables denotes a positive impact, $\mathrm{a}^{\prime}-^{\prime}$ denotes a negative impact, ${ }^{\prime} 0$ ' denotes no impact and ${ }^{\prime}+/{ }^{\prime}$ denotes that the impact can be positive as well as negative, dependent on the specific parameter values. In some cases we have been unable to analytically evaluate the sign of the derivative. In these cases we resort to a numerical analysis and evaluate the impact by taking $\gamma$ fixed to $0.01,0.05,0.1,0.15$, and so on. These cases are marked by a *. We derive some of the results in the tables in the Appendix. We will first discuss the comparative statics results with respect to changes in search costs. The local comparative statics are given in table 1 .

Note that whenever $0<\mu<1$ non-shoppers search less whenever search costs increase. This seems an intuitive result. In general, the table shows that advertising increases in $c$. In the equilibria with full consumer search $(\mu=1)$, the reserve price increases, making it more profitable to advertise. In equilibria with $0<\mu<1$, less consumer search implies that not advertising is less attractive (since a non-advertising firm only attracts those non-shoppers that are searching) and so firms advertise more. In the equilibria where non-shoppers are indifferent between searching and not searching $(0<\mu<1)$, or equivalently where $c$ is relatively high, there is only a single effect of an increase in $c$ on expected prices. The expected nonadvertised price $E p_{0}$ is decreasing in $c$, since in equilibrium $E p_{0}=1-c$. Expected advertised prices also decrease because the increase in advertising leads to more competition. For low $c$, and full consumer search $(\mu=1)$, the expected advertised and non-advertised prices may both increase and decrease. Higher search costs in this equilibrium imply non-shoppers have a higher reservation price. This fact can be exploited by firms to charge higher prices. However, more advertising puts advertised prices under pressure, and since non-advertising firms compete with advertising firms on the shopper segment their prices also tend to decrease. 

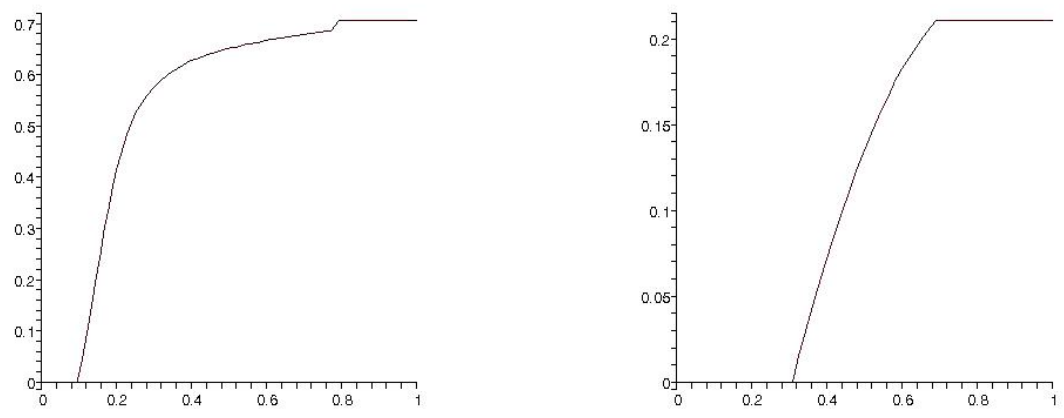

(a) Plots of $\alpha$ as a function of $c$.
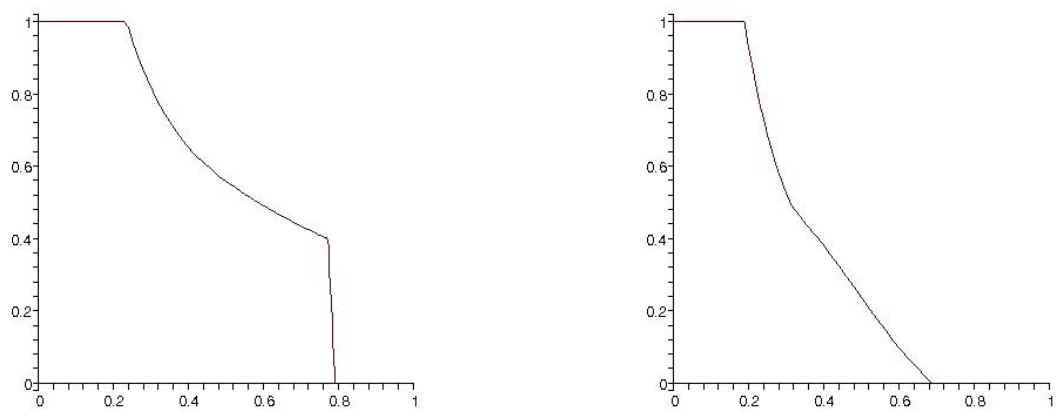

(b) Plots of $\mu$ as a function of $c$.
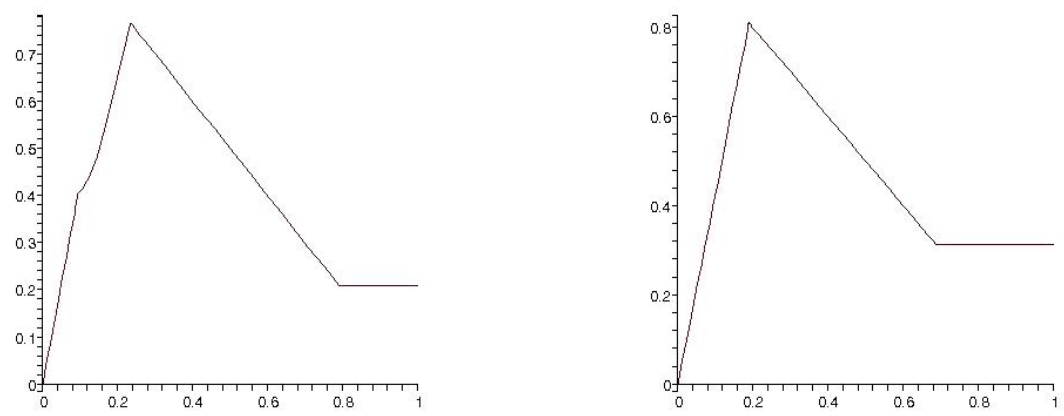

(c) Plots of $E p_{0}$ as a function of $c$.

Figure 3: Plots of several variables as a function of $c$. The left figures are drawn for $\gamma=0.2$ and $A=0.2$, the right figures are drawn for $\gamma=0.2$ and $A=0.6$. 

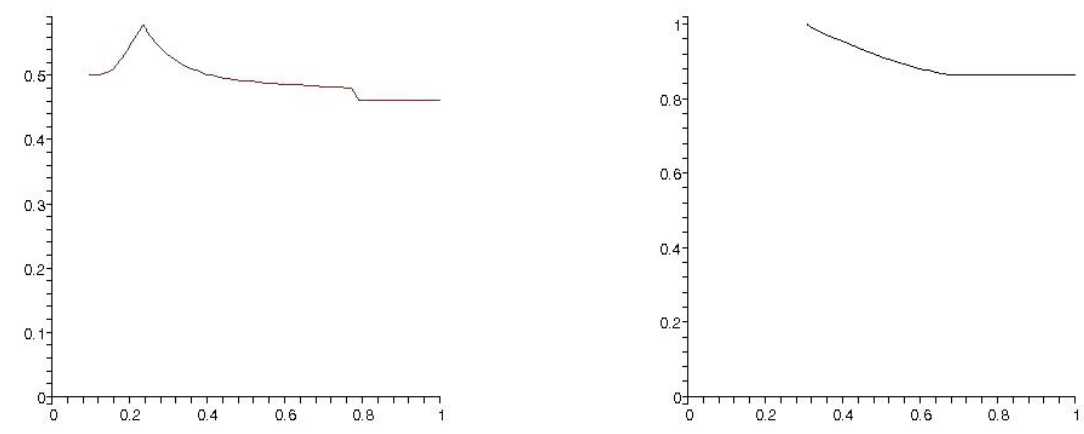

(d) Plots of $E p_{1}$ as a function of $c$.
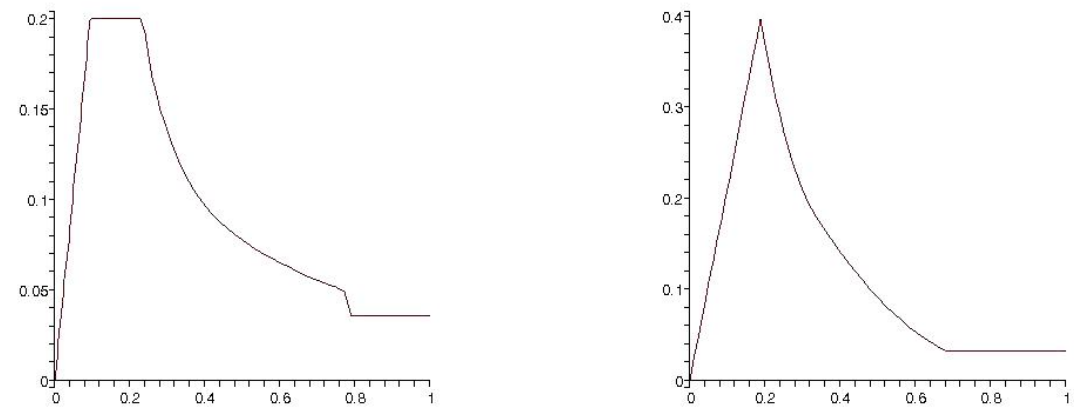

(e) Plots of $\pi$ as a function of $c$.
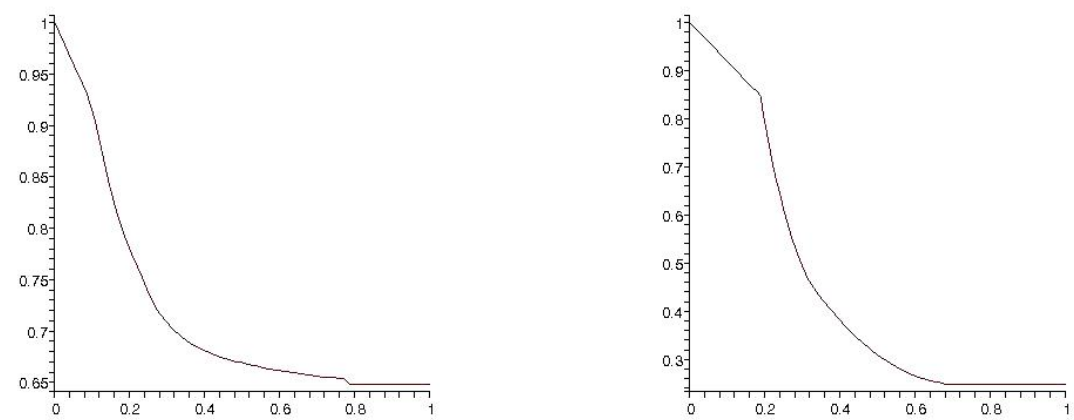

(f) Plots of welfare as a function of $c$.

Figure 3: Plots of several variables as a function of $c$ (continued). The left figures are drawn for $\gamma=0.2$ and $A=0.2$, the right figures are drawn for $\gamma=0.2$ and $A=0.6$. 
An overview of these results is given in figure $3,{ }^{14}$ where we depict $\alpha, \mu$, $E p_{0}, E p_{1}, \pi$ and welfare as a function of $c$, taking $\gamma$ and $A$ fixed. The figures on the left are drawn for $\gamma=0.2$ and $A=0.2$, while the figures on the right are drawn for $\gamma=0.2$ and $A=0.6$. When $A=0.2$, letting $c$ increase from 0 to 1 means that equilibria Ib,IIbi, IIbii, IIaii, II ai, III $a$ and IIIb hold in this order. For $A=0.6$, the figure covers the equilibria Ib, Ia, II $a i, I I I a$ and $I I I b$ in this order. The figure clearly shows how the monotonicity of both $\alpha$ and $\mu$ with respect to changes in $c$ may give rise to non-monotonic behavior of both the expected advertised and non-advertised price with respect to $c$. This non-monotonicity follows from the conflicting forces at work: a higher $c$ implies a higher reservation price creating a tendency for prices to increase, whereas a higher $c$ also implies more advertising and so a higher frequency of price comparing consumers leading to more competition and lower prices. Especially the non-monotonicity of the non-advertised price is striking: when $c$ increases, they may rise from 0 to 0.8 and then fall back again to $0.2-0.4$.

The figure also shows that when search costs increase, profits first increase, but later decrease. Given the behavior of prices and advertising this is quite intuitive. When $c$ is low, prices increase, and so profits increase. However, when $c$ increases further, advertising starts to increase and even though prices increase, the increasing competition resulting from advertising tends to lower profits. The net effect is that profits stay constant. When the search costs are quite high, prices decrease and competition increases, leading to decreasing profits. The figure shows that firms do not unambiguously have an incentive to raise consumer search costs, contradicting claims in the literature (see, e.g., Bakos (1997)) that firms would like search costs to be high to avoid competition.

Welfare decreases in figure 3. There are several effects on welfare. First, with an increase in search costs searching consumers have to pay more and we have seen that more is spent on advertising. These costs work in the direction of a decrease in welfare. Furthermore, there is less consumer search, which means that the non-shoppers who do not receive an ad more often do not buy, which is bad from a welfare point of view as well. On the other hand, more advertising leads on average to more informed non-shoppers, who therefore buy without having to pay search costs. This has a positive effect on welfare. It turns out however that the negative effect on welfare is larger than the positive effect.

Lastly, we note that the primary effect of a change in search costs is twofold. In equilibria with full consumer search, the reservation price increases in the search costs, while in equilibria with partial consumer search $(0<\mu<1)$

\footnotetext{
${ }^{14}$ Note that there are no discontinuities in the graphs and that the scale of the vertical axis can differ per picture. (In the pictures it may look like $E p_{1}$ is discontinuous in $c$. However, when $c$ is small, there is no advertising and so $E p_{1}$ is undefined.)
} 


\begin{tabular}{|c|cccccc|}
\hline & $I a$ & $I b$ & $I I a$ & $I I b$ & $I I I a$ & $I I I b$ \\
\hline$\alpha$ & 0 & 0 & $-^{*}$ & - & $-^{*}$ & - \\
$\mu$ & 0 & 0 & $0 /-$ & 0 & $+/-$ & 0 \\
$E p_{0}$ & 0 & 0 & 0 & $+/ 0$ & 0 & $+/-$ \\
$E p_{1}$ & & & $+^{*}$ & + & $+^{*}$ & + \\
$\pi$ & 0 & 0 & $+/-$ & + & $+/-$ & $+/-$ \\
Welfare & 0 & 0 & $+/-$ & $+/-$ & $+/-$ & - \\
\hline
\end{tabular}

Table 2: Local comparative statics results with respect to changes in A. A * denotes that numerical methods have been used to obtain the result.

the primary effect of a change in search costs is in reducing the amount of search. When the search costs are very high, so that equilibrium IIIb holds, none of $\alpha, \mu, E p_{0}, E p_{1}, \pi$ and welfare depends on $c$. This is because in an equilibrium with such high search costs there is no search at all $(\mu=0)$ and the reservation price exceeds the valuation of the product. Therefore in this case a change in search costs has no effect at all.

We now look at the effects of a change in advertising costs $A$. The impact of a change in advertising costs on the economy is through a change in advertising intensity. If the economy is in an equilibrium without advertising, then a change in advertising costs does not affect the economy at all. If there is advertising, we see the intuitive result that advertising is decreasing in the advertising costs. As a result of less competition in the advertised segment, advertised prices increase in the advertising costs. In case we stay within a region where there is either no search $(\mu=0)$ or full search $(\mu=1)$, there is no impact on search intensity. In the other regions (IIa and III $a$ ) the impact on search intensity is less clear. In equilibrium II $a$ search intensity is defined by the requirement that consumers are indifferent between searching and not searching at all. From equation (6) we see that the price distribution of non-advertised prices over the common support is unaffected by a change in $\alpha$. If this is the distribution that fully captures the behavior of non-advertised prices (case $i i$ ), then the benefits of search are not affected either and therefore the search intensity remains unaffected. If the distribution that describes the behavior of non-advertised prices is also captured by equation (7) (case $i$ ), then it follows that the expected non-advertised price increases due to the fact that the price distribution as defined in equation (7) is increasing in $\alpha$. This implies a reduced incentive to search and a lower search intensity. Similar factors explain the ambiguous impact on search behavior in case of equilibrium IIIa. In this case search intensity and advertising intensity are jointly determined by equations (15) and (16): non-shoppers should be indifferent between searching and not searching and firms should be indifferent between advertising and not advertising. 

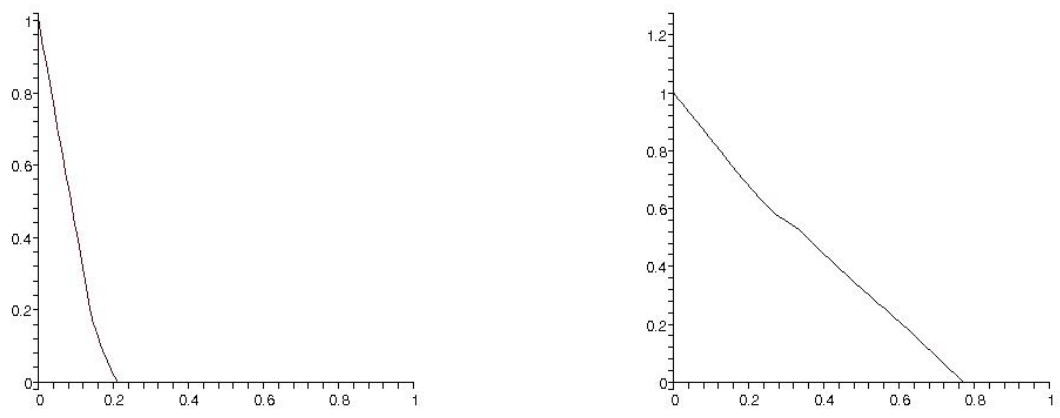

(a) Plots of $\alpha$ as a function of $A$.
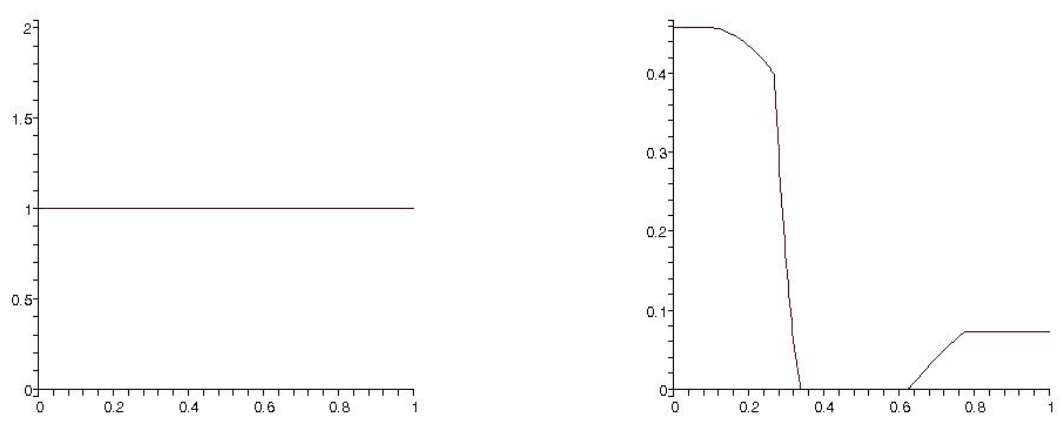

(b) Plots of $\mu$ as a function of $A$.
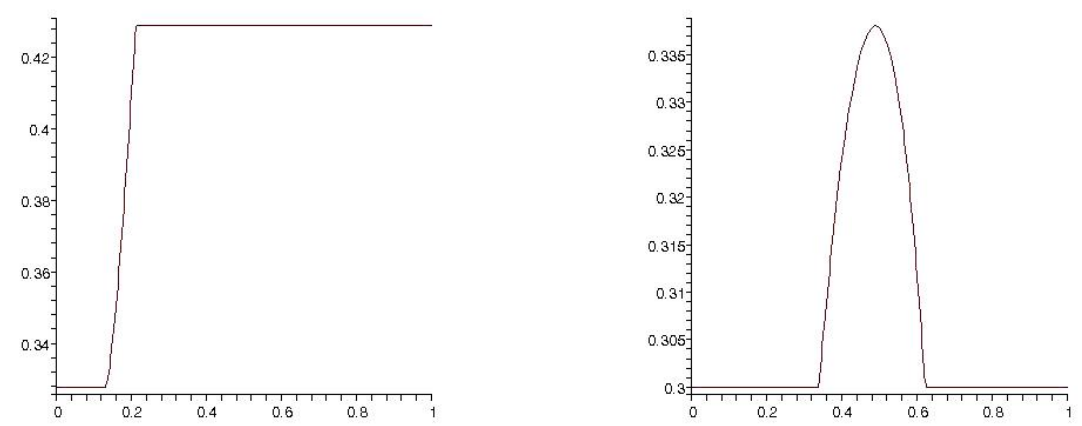

(c) Plots of $E p_{0}$ as a function of $A$.

Figure 4: Plots of several variables as a function of $A$. The left figures are drawn for $\gamma=0.2$ and $c=0.1$, the right figures are drawn for $\gamma=0.2$ and $c=0.7$. 

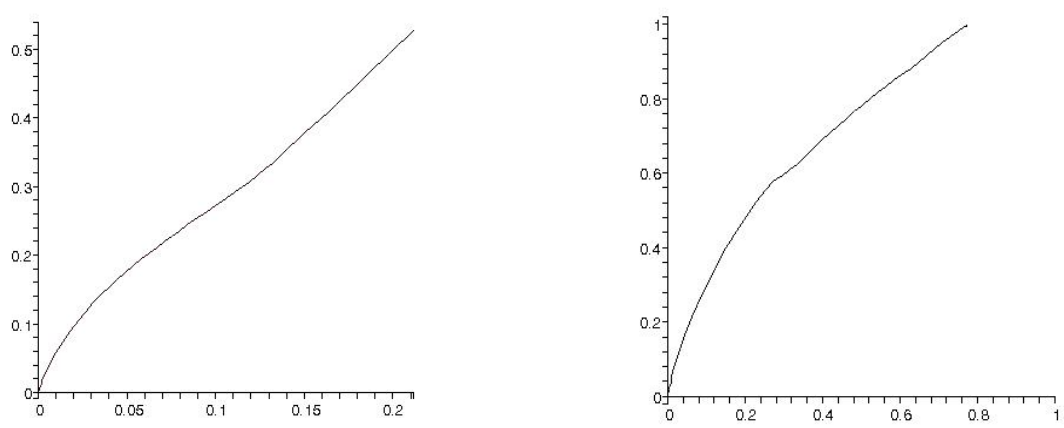

(d) Plots of $E p_{1}$ as a function of $A$.
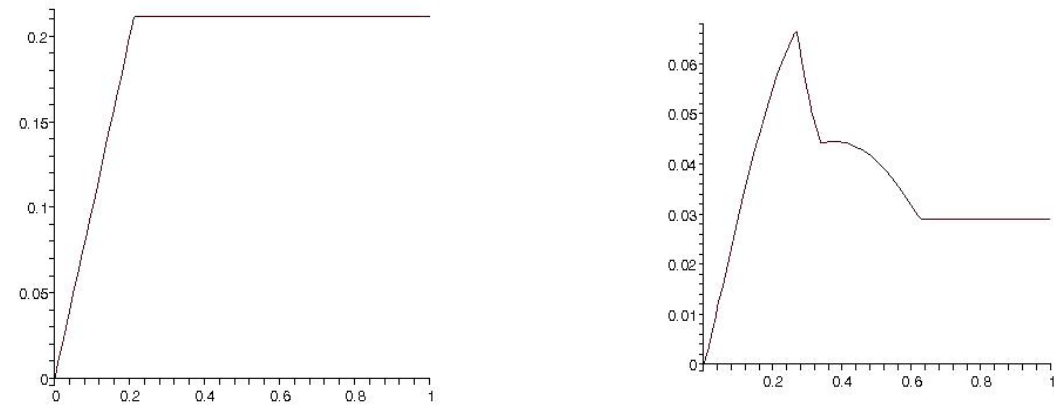

(e) Plots of $\pi$ as a function of $A$.
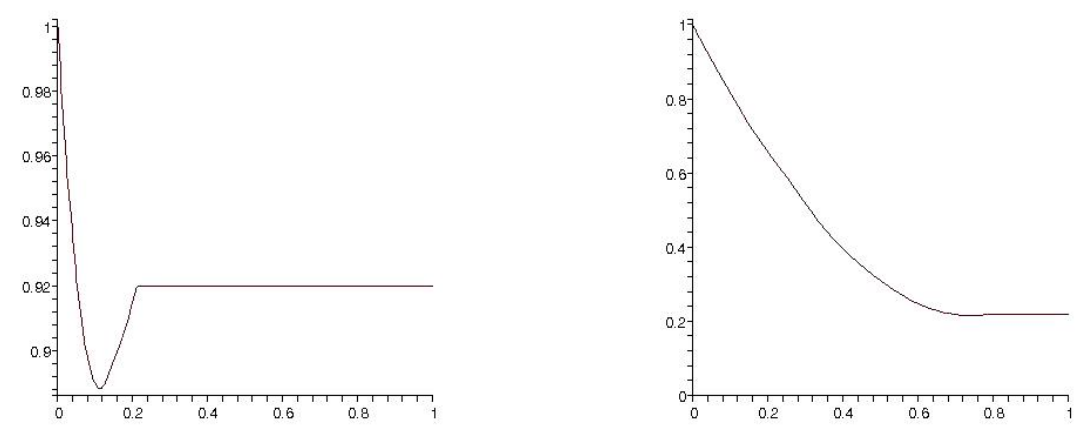

(f) Plots of welfare as a function of $A$.

Figure 4: Plots of several variables as a function of $A$ (continued). The left figures are drawn for $\gamma=0.2$ and $c=0.1$, the right figures are drawn for $\gamma=0.2$ and $c=0.7$. 
The fact that non-advertised prices can both increase, decrease and stay constant in $A$ can be explained as follows. In equilibria $I I a$ and IIIa the expected non-advertised prices cannot change since in these equilibria consumers should be indifferent between search and no search. In equilibria $I I b$ and $I I I b$ the expected non-advertised prices however can change. How this change works out precisely is easiest to see in case of equilibrium $I I I b$. In this equilibrium $\mu=0$ so non-advertising firms only sell to shoppers. Furthermore, advertised prices are always higher than non-advertised prices so if a non-advertising firm competes with an advertising firm, the nonadvertising firm attracts all the shoppers. The effect of an increase in $A$ and the corresponding decrease in advertising is twofold. The minimum advertised price rises, making it possible for non-advertised prices to rise since there is no gap between $F_{0}(p)$ and $F_{1}(p)$. On the other hand a lower probability that the competitor advertises lowers the probability of having monopoly power on the shopper segment, thus lowering the expected non-advertised price. The net effect can be both positive and negative. In equilibrium type $I I b$, these two effects play a role, but there is an additional effect since a non-advertising firm in this equilibrium also attracts searching non-shoppers who do not receive an ad. When the probability of advertising decreases the probability of attracting these consumers increases and since a firm has monopoly power on them the non-advertised price can increase. An overview of these results is depicted in figure 4, where $\alpha, \mu, E p_{0}, E p_{1}, \pi$ and welfare are represented as a function of $A$, taking $\gamma$ and $c$ fixed. In the left graphs, we take $\gamma=0.2$ and $c=0.1$, while in the right graphs $\gamma=0.2$ and $c=0.7$. When $c=0.1$, letting $A$ increase from 0 to 1 means that equilibria $I I b i i, I I b i$ and $I b$ hold in this order. For $c=0.7$ equilibria II aii, IIai, III a III b, III a and Ia hold in this order. Note that for $c=0.1$ we plot $E p_{1}$ only for $A<0.21$. For higher values of $A E p_{1}$ is not defined. The figures show that although $E p_{0}$ is non-monotonic in $A$, the expected non-advertised price has only little variation. This is in contrast with the expected advertised price, which monotonically increases from 0 to $0.5-1$. Profits and welfare can both both increase and decrease in the advertising costs. The effect on profits can be explained by the fact that an increase in advertising costs implies on one hand less competition and therefore higher advertised prices, but on the other hand also leads to more expenditure on advertising if firms decide to advertise. The ambiguous effect on welfare is already apparent from the advertising term in the expression for welfare, as advertising decreases while the costs increase and the net effect can be both positive and negative.

We finally look at the effect of an increase in the fraction of shoppers $\gamma$. When the number of shoppers increases, it is clear that the advertising intensity decreases as ads are less effective: advertisements only affect the nonshoppers, who get informed through ads, and the fraction of non-shoppers 


\begin{tabular}{|c|cccccc|}
\hline & $I a$ & $I b$ & $I I a$ & $I I b$ & $I I I a$ & $I I I b$ \\
\hline$\alpha$ & 0 & 0 & $-^{*}$ & - & $-^{*}$ & - \\
$\mu$ & + & 0 & $+/-$ & 0 & $+/-$ & 0 \\
$E p_{0}$ & 0 & - & 0 & $+/-$ & 0 & $+/-$ \\
$E p_{1}$ & & & $+^{*}$ & $+/-$ & $+^{*}$ & $+/-$ \\
$\pi$ & + & - & $+/-$ & 0 & $+/-$ & $+/-$ \\
Welfare & + & + & $+/-$ & $+/-$ & $+/-$ & $+/-$ \\
\hline
\end{tabular}

Table 3: Local comparative statics results with respect to changes in $\gamma$. A * denotes that numerical methods have been used to obtain the result.

decreases. Expected prices can both increase and decrease in $\gamma$. More shoppers leads to more competition and lower prices, but less advertising leads to less competition and higher prices. These two effects can lead to both a net increase and to a net decrease in prices. The resulting profits for firms can also both increase and decrease in $\gamma$ and the same holds for welfare. Here, when there are more shoppers, less consumers have to pay search costs or do not buy at all, which has a positive effect on welfare. But the resulting decrease in advertising implies that less non-shoppers are informed, which is bad from a welfare point of view. The resulting welfare change can be both positive and negative. Again, it is not the case that firms should necessarily lose out when consumers use more and more search engines as the direct pro-competitive effect could be entirely offset by a decrease in advertising expenditures and a resulting incentive to increase advertised prices.

\section{Conclusion}

In this paper we have analyzed the interaction between two information transferring technologies: advertising and search. In particular we have focused on the interaction between the incentives for firms to advertise and for consumers to search in relation to the parameters underlying the search and advertising technology. We take the view that an important role of advertising is not just to inform consumers of the price of the product, but especially of the fact that the firm (shop) carries the product under consideration so that after receiving an ad from the shop a consumer is no longer uncertain whether the shop carries the product at all. An implication of this view is that the search cost of consumers visiting a shop from which they have received an ad is much lower than the search cost of visiting a shop from which no advertisement is received. In the paper we analyze the extreme case where this search cost after receiving an ad is negligibly small. Apart from this interpretation of advertising, the advertising technology is simply characterized by a single parameter indicating the constant marginal 
cost of reaching one more consumer. On the consumer side, there are two types of agents: (a small fraction of) shoppers who are fully informed about prices at no cost and other consumers who search with a standard sequential search protocol with constant search cost. By studying the implications of this set of assumptions, our analysis is complementary to that of the seminal paper by Robert and Stahl (1993).

In this set-up, we reach some important somewhat controversial conclusions. First, if the cost of one of these technologies becomes arbitrary small, the model's outcome get very close to the fully competitive outcome where price equals marginal cost. The fully competitive outcome occurs when consumers are somehow fully informed of all prices. This will necessarily happen if one of the technologies becomes arbitrarily cheap. Second, there are important non-monotonicities in the relations between expected prices on one hand and search and advertising cost on the other hand. This means that it may very well be the case that when, for example, search cost decrease expected price increase. An important part of the explanation for this phenomenon to occur is that search and advertising are 'substitutes' over an important domain of the model's parameters in the sense that if consumers (initially) start searching more (because of a decrease in search cost) firms will advertise less. In the aggregate this may mean that consumers actually are less informed about prices when search cost are lower and this gives firms an incentive to raise prices. Third, advertised prices may actually be higher than nonadvertised prices for some region of the parameter space (for example when the advertising cost are not too low and the search cost is relatively high). This happens, for example, when there is relatively little search so that most consumers that consider buying from non-advertising firms are shoppers. As these consumers are fully informed about prices, this forces these firms to charge quite low prices. Advertising firms will focus then on the nonshoppers and as they will not search further if they have received an ad, these firms enjoy some monopoly power if there is not too much advertising in the economy. 


\section{Appendix A}

\section{Proof of Lemma 3.3}

Suppose $F_{0}(p)$ has an atom at $\hat{p}$ and consider a firm not advertising and charging a price $\hat{p}^{15}$ We will show that this firm has an incentive to lower its price slightly.

Suppose the competitor also does not advertise and charges price $\hat{p}$, what happens with nonzero probability. Then the per-firm profits are

$$
\hat{p}\left[\frac{1}{2} \gamma+\frac{1}{2}(1-\gamma) I_{r_{0}<\theta}+\mu \frac{1}{2}(1-\gamma) I_{r_{0}=\theta}\right],
$$

where $I_{r_{0}<\theta}$ is 1 if $r_{0}<\theta$ and 0 otherwise.

If the firm decides to set price $\hat{p}-\varepsilon$ it makes profit

$$
(\hat{p}-\varepsilon)\left[\gamma+(1-\gamma) I_{r_{0}<\theta}\left(\frac{1}{2} I_{\hat{p} \leq r_{0}}+I_{\hat{p}>r_{0}}\right)+\mu \frac{1}{2}(1-\gamma) I_{r_{0}=\theta}\right],
$$

which is larger than the expression above provided $\varepsilon$ is small. (Recall from lemma 3.1 that $\hat{p}>0$ so also $\hat{p}-\varepsilon>0$ for $\varepsilon$ small.)

Suppose the competitor chooses another strategy. Lowering the price charged will never reduce the number of sales. The loss from lowering the price by $\varepsilon$ will therefore at most be $\varepsilon$.

Lowering the price by $\varepsilon$ will with positive probability lead to a gain and with the complementary probability lead to a loss of at most $\varepsilon$. Choosing $\varepsilon$ small enough will lead to a net gain. This shows firms have an incentive to charge $\hat{p}-\varepsilon$ instead of $\hat{p}$, which is a contradiction.

We will now prove the fact that $F_{0}\left(r_{0}\right)=F_{1}\left(r_{0}\right)=1$. Suppose $\bar{p}_{0} \geq \bar{p}_{1}$ and $\bar{p}_{0}>r_{0}$ Then a firm not advertising and charging $\bar{p}_{0}$ does not sell any item and makes profit 0 . This contradicts the fact that expected profits are strictly positive, as explained below lemma 3.2.

Suppose $\bar{p}_{0} \leq \bar{p}_{1}$ and $\bar{p}_{1}>r_{0}$. Then a firm advertising and charging $\bar{p}_{1}$ does not sell any item and makes profit $-A$. This again contradicts the corollary on strictly positive profits.

\section{Proof of Lemma 3.4}

We will here prove the part of the theorem about $\pi_{0}(p)$. The proof for $\pi_{1}(p)$ follows the same lines and will be skipped. We first note that for all $p$ in the support of $F_{0}(p), \pi_{0}(p)=\pi_{0}$ by definition of an equilibrium. Furthermore, note that $\pi_{0}(p)=p\left(\alpha \gamma\left(1-F_{1}(p)\right)+\frac{1}{2}(1-\alpha)(1-\gamma) \mu+(1-\alpha) \gamma\left(1-F_{0}(p)\right)\right)$ and $\pi_{1}(p)=p\left(\alpha\left(1-F_{1}(p)\right)+(1-\alpha)(1-\gamma)+(1-\alpha) \gamma\left(1-F_{0}(p)\right)\right)-A$. Since there are no atoms in the price distributions (see lemma 3.3), both profit functions are continuous. We also note that since $\underline{p}_{0}$ and $\bar{p}_{0}$ are both in the support of $F_{0}(p), \pi_{0}\left(\underline{p}_{0}\right)=\pi_{0}\left(\bar{p}_{0}\right)=\pi_{0}$. We now proceed the argument

\footnotetext{
${ }^{15}$ The proof that $F_{1}(p)$ is atomless follows a similar argument and will be skipped.
} 
by contradiction. Suppose $\pi_{0}(p)$ is not constant. Then there is at least one price $\hat{p}$ for which $\pi_{0}(\hat{p}) \neq \pi_{0}$. If $\pi_{0}(\hat{p})>\pi_{0}$, setting price $\hat{p}$ would be better than setting price $\underline{p}_{0}$, contradicting the fact that $\underline{p}_{0}$ is in the support of $F_{0}(p)$. If $\pi_{0}(\hat{p})<\pi_{0}, \hat{p}$ is not in the support of $F_{0}(p)$ and due to the continuity of $\pi_{0}(p)$ it is possible to find $\underline{p}$ and $\bar{p}$, with $\underline{p}<\hat{p}<\bar{p}$ such that $\pi_{0}(\underline{p})=\pi_{0}(\bar{p})=\pi_{0}$ and for every $0<x<1, \pi_{0}\left(p^{*}\right)=\pi_{0}(x \underline{p}+(1-x) \bar{p})<$ $\pi_{0}=x \pi_{0}(p)+(1-x) \pi_{0}(\bar{p})$. Using the above expression for $\pi_{0}(p)$, and the fact that for all $0<x<1 p^{*}$ is not in the support of $F_{0}(p)$, this can be rewritten as $p^{*}\left(1-F_{1}\left(p^{*}\right)\right)<x p\left(1-F_{1}(p)\right)+(1-x) \bar{p}\left(1-F_{1}(\bar{p})\right)$. Using this we can show that $\pi_{1}\left(p^{*}\right)<x \pi_{1}(\underline{p})+(1-x) \pi_{1}(\bar{p})$ and so no advertising firm will ever charge $p^{*}$. However, since $p^{*}$ is also not in the support of $F_{0}(p)$, both price distributions have a gap in the price region $(\underline{p}, \bar{p})$. This gives $\pi_{0}(\underline{p})<\pi_{0}(\bar{p})$, a contradiction.

\section{Proof of Theorem 3.5}

There are three possibilities for an equilibrium not to be of one of the types mentioned in the theorem. We will show that all these three possibilities cannot be part of an equilibrium.

$0<\alpha<1$ and $\bar{p}_{0}>\bar{p}_{1}$ Using standard techniques, we note that in this case $\bar{p}_{0}=\min \left\{r_{0}, 1\right\}$ and that $F_{0}(p)=1-\frac{\left(\bar{p}_{0}-p\right) \frac{1}{2} \mu(1-\gamma)}{p \gamma}$ for $p \geq \bar{p}_{1}$. Whenever $p>\bar{p}_{1}$, the profit for an advertising firm equals $\pi_{1}(p)=$ $p\left[\gamma(1-\alpha)\left(1-F_{0}(p)\right)+(1-\gamma)(1-\alpha)\right]-A$. Using the expression for $F_{0}(p)$ it can be shown that

$$
\pi_{1}(p)=(1-\alpha) \frac{1}{2} \mu(1-\gamma)\left(\bar{p}_{0}-p\right)+(1-\gamma)(1-\alpha) p-A,
$$

which is increasing in $p$ for $\bar{p}_{1} \leq p \leq \bar{p}_{0}$, showing that advertising firms have an incentive to deviate.

$0<\alpha<1$ and $\underline{p}_{1}<\bar{p}_{0}<\bar{p}_{1}$ In this case, using lemma 3.4, $\pi_{0}(p)=\pi_{1}(p)$ in the price region $\left[\max \left\{\underline{p}_{0}, \underline{p}_{1}\right\}, \bar{p}_{0}\right]$, giving an expression for $F_{1}(p)$ as follows:

$$
F_{1}(p)=1-\frac{A-\left(1-\frac{1}{2} \mu\right) p(1-\alpha)(1-\gamma)}{\alpha(1-\gamma) p}
$$

Moreover, using this and $\pi_{0}\left(\bar{p}_{0}\right)=\pi_{0}(p)$ we get $F_{0}(p)=1-\frac{\left(\bar{p}_{0}-p\right)\left(\frac{1}{2} \mu-\gamma\right)}{p \gamma}$ on $\left[\max \left\{\underline{p}_{0}, \underline{p}_{1}\right\}, \bar{p}_{0}\right]$. To make sure $F_{0}(p)$ is below $1, \frac{1}{2} \mu-\gamma$ should be positive. We furthermore can derive an expression for $F_{1}(p)$ for $p \geq \bar{p}_{0}$ by using $\pi_{1}(p)=\pi_{1}\left(\bar{p}_{0}\right)$, which can be written as 
$p\left[\left(1-F_{1}(p)\right)+(1-\gamma)(1-\alpha)\right]-A=\bar{p}_{0}\left[\left(1-F_{1}\left(\bar{p}_{0}\right)\right)+(1-\gamma)(1-\alpha)\right]-A$.

This gives for $p \geq \bar{p}_{0}$,

$$
1-F_{1}(p)=\frac{\alpha \bar{p}_{0}\left[\left(1-F_{1}\left(\bar{p}_{0}\right)\right]-(1-\alpha)(1-\gamma)\left(p-\bar{p}_{0}\right)\right.}{\alpha p}
$$

Plugging this expression into $\pi_{0}(p)$ for $p \geq \bar{p}_{0}$ gives $\pi_{0}(p)=\gamma \bar{p}_{1}(1-$ $\alpha)(1-\gamma)+p(1-\alpha)(1-\gamma)\left(\frac{1}{2} \mu-\gamma\right)$, which is increasing in $p$ since $\frac{1}{2} \mu-\gamma$ is positive. This shows the non-advertising firms have an incentive to deviate.

$0<\alpha<1$ and $\bar{p}_{0}<\underline{p}_{1}$ In this case, $\pi_{0}(p)=p\left(\alpha \gamma+(1-\alpha)(1-\gamma) \frac{1}{2} \mu\right)$ for $\bar{p}_{0} \leq p \leq \underline{p}_{1}$, which is increasing in $p$ and so non-advertising firms have an incentive to deviate.

\section{Remainder of the Proof of Proposition 3.7}

Equilibrium type $b$ assumes $\mu=1$. The other main difference with equilibrium $I a$ is that now $r_{0}<1$ instead of $r_{0}=1$. The equilibrium can be derived along the same lines as equilibrium $I a$ and gives the same expression for $F_{0}(p)$, except that now $\mu=1$. Using $\int_{\underline{p}_{0}}^{r_{0}} F_{0}(p) d p=c$ we derive that $r_{0}=\frac{c}{1+\frac{1-\gamma}{2 \gamma} \ln \frac{1-\gamma}{1+\gamma}}=\frac{c}{\beta_{1}}$. Note that $r_{0}<1$ gives restriction

$$
c<\beta_{1} \text {. }
$$

Furthermore, for $\alpha=0$ to hold, it should not be profitable to deviate to advertising. This, like in equilibrium $I a$, gives restriction

$$
(1-\gamma) r_{0}-A<\frac{1}{2}(1-\gamma) r_{0} \Leftrightarrow c<\frac{2 A \beta_{1}}{1-\gamma} .
$$

\section{Remainder of the Proof of Proposition 3.8}

We first continue the derivation of the conditions under which equilibrium II $a$ holds. We define $g(x)=1+\frac{1-\gamma}{2 \gamma} x \ln \frac{\frac{1}{2} x A}{\frac{1}{2} x A+\left(1-\frac{1}{2} x\right) \gamma}-\left(1-\frac{1}{2} x\right) \ln \frac{A\left(1-\frac{1}{2} x\right)}{(1-\gamma)\left(1-\frac{1}{2} x\right)-\frac{1}{2} A x}$.

We also define $f(x)=1-\frac{\gamma-\frac{1}{2} x}{\gamma} \ln \frac{x-2 \gamma}{x}$. Note that $g(\mu)=c$ defines $\mu$ for equilibrium IIai and that $f(\mu)=c$ defines $\mu$ for equilibrium IIaii. We furthermore note that $f(x)$ and $g(x)$ are decreasing in $x$, the proof is available on request. We also define $x_{1}=1+\gamma-A-\sqrt{(1+\gamma-A)^{2}-4 \gamma}$ and $x_{2}=1+\gamma-A+\sqrt{(1+\gamma-A)^{2}-4 \gamma}$ and note that $g\left(x_{1}\right)=f\left(x_{1}\right)$ and $g\left(x_{2}\right)=f\left(x_{2}\right)$. 
Note that for restriction (11) to make sense, $\underline{p}_{0}$ should be smaller than $\underline{p}_{1}$. This restriction gives $\frac{\frac{1}{2} \mu r_{0} A}{\frac{1}{2} \mu A+\left(1-\frac{1}{2} \mu\right) \gamma r_{0}}<\frac{A}{(1-\gamma)\left(1-\frac{1}{2} \mu(1-\alpha)\right)}$, which can be rewritten as $\frac{1}{2} \mu r_{0}(1-\gamma)\left(1-\frac{1}{2} \mu(1-\alpha)\right)<\frac{1}{2} \mu A+\left(1-\frac{1}{2} \mu\right) \gamma r_{0}$. Using expression (4), and $r_{0}=1$, this can be rewritten as $\left(1-\frac{1}{2} \mu\right)(2 \gamma-\mu)+A \mu>0$ or $\frac{1}{2} \mu^{2}-(1+\gamma-A) \mu+2 \gamma>0$. This function is a quadratic function with zero points at $\mu=1+\gamma-A \pm \sqrt{(1+\gamma-A)^{2}-4 \gamma}$, or lies completely above the x-axis if $(1+\gamma-A)^{2}-4 \gamma<0$. This means that $\underline{p}_{0}<\underline{p}_{1}$ if and only if $(1+\gamma-A)^{2}-4 \gamma<0$ or $\mu<1+\gamma-A-\sqrt{(1+\gamma-A)^{2}-4 \gamma}$ or $\mu>1+\gamma-A+\sqrt{(1+\gamma-A)^{2}-4 \gamma}$. Note that $(1+\gamma-A)^{2}-4 \gamma<0$ can be rewritten as $A^{2}-2 A(1+\gamma)+(1-\gamma)^{2}<0$ or $\frac{(1-\gamma)^{2}}{(1+\sqrt{\gamma})^{2}}<A<\frac{(1-\gamma)^{2}}{(1-\sqrt{\gamma})^{2}}$.

Now note that for the equilibrium to exist, $2-\frac{2 A}{(1-\gamma)}$ should be larger than $2 \gamma$, since $2 \gamma<\mu<2-\frac{2 A}{(1-\gamma)}$. This gives $A<(1-\gamma)^{2}$. Since $\frac{(1-\gamma)^{2}}{(1-\sqrt{\gamma})^{2}}>(1-\gamma)^{2}$, whenever $A>\frac{(1-\gamma)^{2}}{(1+\sqrt{\gamma})^{2}}$ we are done with the restriction $\underline{p}_{0}<\underline{p}_{1}$ and we only have to deal with restriction (11). When $A<\frac{(1-\gamma)^{2}}{(1+\sqrt{\gamma})^{2}}$, we have the extra restriction that either $\mu<1+\gamma-A-\sqrt{(1+\gamma-A)^{2}-4 \gamma}$ or $\mu>1+\gamma-A+\sqrt{(1+\gamma-A)^{2}-4 \gamma}$. This can be translated in $c>g\left(x_{1}\right)$ or $c<g\left(x_{2}\right)$ by using the definition of $\mu$ given by expression (9).

For restriction (12) to make sense, $\underline{p}_{0}>\underline{p}_{1}$ should hold. This restriction gives $\frac{1}{2} \mu^{2}-(1+\gamma-A) \mu+2 \gamma<0$. This means that $\underline{p}_{0}>\underline{p}_{1}$ if and only if $(1+\gamma-A)^{2}-4 \gamma>0$ and $\mu>1+\gamma-A-\sqrt{(1+\gamma-A)^{2}-4 \gamma}$ and $\mu<1+\gamma-A+\sqrt{(1+\gamma-A)^{2}-4 \gamma}$. Note that $(1+\gamma-A)^{2}-4 \gamma>0$ can be rewritten as $A<\frac{(1-\gamma)^{2}}{(1+\sqrt{\gamma})^{2}}$ or $A>\frac{(1-\gamma)^{2}}{(1-\sqrt{\gamma})^{2}}$. Again, $2-\frac{2 A}{(1-\gamma)}$ should be larger than $2 \gamma$, and so $A>\frac{(1-\gamma)^{2}}{(1-\sqrt{\gamma})^{2}}$ is not possible. Therefore, equilibrium type IIaii exists when restriction (12) is met, and $A<\frac{(1-\gamma)^{2}}{(1+\sqrt{\gamma})^{2}}$ and $1+\gamma-A-\sqrt{(1+\gamma-A)^{2}-4 \gamma}<\mu<1+\gamma-A+\sqrt{(1+\gamma-A)^{2}-4 \gamma}$. Note that $1+\gamma-A-\sqrt{(1+\gamma-A)^{2}-4 \gamma}<\mu<1+\gamma-A+\sqrt{(1+\gamma-A)^{2}-4 \gamma}$ can be rewritten as $f\left(x_{2}\right)<c<f\left(x_{1}\right)$ by using definition (10).

Combining the above results, and noting that restriction (11) can be rewritten as $\max \left\{g(1), g\left(2-\frac{2 A}{1-\gamma}\right)\right\}<c<g(2 \gamma)$ and restriction (12) can be rewritten as $\max \left\{f(1), f\left(2-\frac{2 A}{1-\gamma}\right)\right\}<c<f(2 \gamma)$ we get that equilibrium IIa exists for $A<\frac{(1-\gamma)^{2}}{(1+\sqrt{\gamma})^{2}}$ whenever

$$
\max \left\{g\left(x_{1}\right), g(1), g\left(2-\frac{2 A}{1-\gamma}\right)\right\}<c<g(2 \gamma)
$$

or 


$$
\max \left\{f(1), f\left(2-\frac{2 A}{1-\gamma}\right), f\left(x_{2}\right)\right\}<c<\min \left\{f\left(x_{1}\right), f(2 \gamma)\right\}
$$

or

$$
\max \left\{g(1), g\left(2-\frac{2 A}{1-\gamma}\right)\right\}<c<\min \left\{g\left(x_{2}\right), g(2 \gamma)\right\}
$$

and exists for $A>\frac{(1-\gamma)^{2}}{(1+\sqrt{\gamma})^{2}}$ whenever restriction (11) holds.

Note that since $g(x)$ is decreasing in $x, \max \left\{g(1), g\left(2-\frac{2 A}{1-\gamma}\right), g\left(x_{1}\right)\right\}=$ $g\left(\min \left\{1,2-\frac{2 A}{1-\gamma}, x_{1}\right\}\right)$ and using that $f(x)$ is decreasing in $x, \max \left\{f\left(x_{2}\right), f(1), f(2-\right.$ $\left.\left.\frac{2 A}{1-\gamma}\right)\right\}=f\left(\min \left\{x_{2}, 1,2-\frac{2 A}{1-\gamma}\right\}\right)$.

We now first assume $A<\frac{1-2 \gamma}{2}<\frac{(1-\gamma)^{2}}{(1+\sqrt{\gamma})^{2}}$. Under this assumption, $2-\frac{2 A}{1-\gamma}>$ $1, x_{1}<1$ (using that $x_{1}$ is increasing in $A$ ) and $x_{2}>1$ (using that $x_{2}$ is decreasing in $A)$. This last inequality means that $g\left(x_{2}\right)<g(1)$ and so $\max \left\{g(1), g\left(2-\frac{2 A}{1-\gamma}\right)\right\}<c<\min \left\{g\left(x_{2}\right), g(2 \gamma)\right\}$ is not possible. Using that $x_{1}$ is increasing in $A$, it is easy to see that $x_{1}>2 \gamma$ and so we can rewrite the parameter restrictions above as $g\left(x_{1}\right)<c<g(2 \gamma)$ or $\beta_{2}=f(1)<c<$ $f\left(x_{1}\right)=g\left(x_{1}\right)$. This finally gives parameter restrictions $\beta_{2}<c<g(2 \gamma)=$ $1+(1-\gamma) \ln \frac{(1-\gamma)^{2}-A \gamma}{A(1-\gamma)+(1-\gamma)^{2}}$ for $A<\frac{1-2 \gamma}{2}$.

Now assume $\frac{1-2 \gamma}{2}<A<\frac{(1-\gamma)^{2}}{(1+\sqrt{\gamma})^{2}}$. In this case, $x_{2}>2 \gamma$ and $x_{1}<2-\frac{2 A}{1-\gamma}$. When $\gamma<\frac{1}{4}, x_{1}<1, x_{2}<1$ and $x_{2}<2-\frac{2 A}{1-\gamma}$. So for $\gamma<\frac{1}{4}$, the region where equilibrium $I I a$ holds can be written as $g\left(x_{1}\right)<c<g(2 \gamma)$ or $f\left(x_{2}\right)<c<f\left(x_{1}\right)$ or $\max \left\{g(1), g\left(2-\frac{2 A}{1-\gamma}\right)\right\}<c<g\left(x_{2}\right)$, what can be combined as $\max \left\{g(1), g\left(2-\frac{2 A}{1-\gamma}\right)\right\}<c<g(2 \gamma)$. When $\gamma>\frac{1}{4}, x_{2}>1$ and $x_{1}>1$, and so $\max \left\{f\left(x_{2}\right), f(1), f\left(2-\frac{2 A}{1-\gamma}\right)\right\}<c<\min \left\{f\left(x_{1}\right), f(2 \gamma)\right\}$ and $\max \left\{g(1), g\left(2-\frac{2 A}{1-\gamma}\right)\right\}<c<\min \left\{g\left(x_{2}\right), g(2 \gamma)\right\}$ are both empty regions. Equilibrium IIa in this case exists for $\max \left\{g(1), g\left(2-\frac{2 A}{1-\gamma}\right), g\left(x_{1}\right)\right\}<c<$ $g(2 \gamma)$, or, since $x_{1}>1, \max \left\{g(1), g\left(2-\frac{2 A}{1-\gamma}\right)\right\}<c<g(2 \gamma)$. Therefore, when $\frac{1-2 \gamma}{2}<A<\frac{(1-\gamma)^{2}}{(1+\sqrt{\gamma})^{2}}$, equilibrium II a exists when $\max \left\{1+\frac{1-\gamma}{2 \gamma} \ln \frac{A}{A+\gamma}-\right.$ $\left.\frac{1}{2} \ln \frac{A}{1-\gamma-A}, 1+\frac{1-\gamma-A}{\gamma} \ln \frac{1-\gamma-A}{1-A}\right\}<c<1+(1-\gamma) \ln \frac{(1-\gamma)^{2}-A \gamma}{A(1-\gamma)+(1-\gamma)^{2}}$.

As already mentioned before, when $A>\frac{(1-\gamma)^{2}}{(1+\sqrt{\gamma})^{2}}$, equilibrium IIa holds when $\max \left\{1+\frac{1-\gamma}{2 \gamma} \ln \frac{A}{A+\gamma}-\frac{1}{2} \ln \frac{A}{1-\gamma-A}, 1+\frac{1-\gamma-A}{\gamma} \ln \frac{1-\gamma-A}{1-A}\right\}<c<1+(1-$ $\gamma) \ln \frac{(1-\gamma)^{2}-A \gamma}{A(1-\gamma)+(1-\gamma)^{2}}$.

This shows that the parameter restrictions for equilibrium $I I a$ are as in Proposition 3.8 .

We now proceed to the derivation of equilibrium $I I b$. This derivation follows the same lines as the derivation of equilibrium IIa. Again, there are two types of equilibrium: type $i$ with $\underline{p}_{0}<\underline{p}_{1}$ and type $i i$ with $\underline{p}_{0}>\underline{p}_{1}$. We get 
the same expressions for $F_{0}(p), F_{1}(p)$ and $\alpha$ as for equilibrium IIa, except that now $\mu=1$ and $r_{0}<1$.

For case $i, \int_{\underline{p}_{0}}^{r_{0}} F_{0}(p) d p=c$, implicitly defines $r_{0}$ as $r_{0}\left(1+\frac{1}{2} \ln \left(\frac{r_{0}}{A}(1-\gamma)-\right.\right.$ $\left.1)-\frac{1-\gamma}{2 \gamma} \ln \left(\frac{r_{0}}{A} \gamma+1\right)\right)=c$. Note that for $\frac{A}{1-\gamma}<r_{0}<\frac{2 A}{1-2 \gamma}$ this expression is increasing in $r_{0}{ }^{16}$.

For equilibrium $I I b i$ to exist, we need that $0<\alpha<1$, what translates to $r_{0}>\frac{2 A}{1-\gamma}$. Furthermore, $\underline{p}_{0}<\underline{p}_{1}$ gives restriction $r_{0}<\frac{2 A}{1-2 \gamma}$. And finally, $r_{0}<1$. These three restrictions can be expressed in terms of $c$ and $A$ using the implicit definition of $r_{0}$. This gives

$$
\frac{2 A \beta_{1}}{1-\gamma}<c<\frac{2 A \beta_{2}}{1-2 \gamma}
$$

for $A<\frac{1-2 \gamma}{2}$, and

$$
\frac{2 A \beta_{1}}{1-\gamma}<c<1+\frac{1}{2} \ln \left(\frac{1-\gamma}{A}-1\right)-\frac{1-\gamma}{2 \gamma} \ln \left(\frac{\gamma}{A}+1\right)
$$

for $A>\frac{1-2 \gamma}{2}$.

For case $i i, \int_{\underline{p}_{0}}^{r_{0}} F_{0}(p) d p=c$ gives $r_{0}=\frac{c}{1+\frac{\frac{1}{2}-\gamma}{\gamma} \ln (1-2 \gamma)}=\frac{c}{\beta_{2}}$. The equilibrium exists when $r_{0}>\frac{2 A}{1-2 \gamma}$ (required because $\underline{p}_{0}>\underline{p}_{1}$ is assumed), and again $\frac{2 A}{1-\gamma}<r_{0}<1$. Note that $r_{0}>\frac{2 A}{1-\gamma}$ holds as long as $r_{0}>\frac{2 A}{1-2 \gamma}$ holds. This gives parameter restrictions

$$
\frac{2 A \beta_{2}}{1-2 \gamma}<c \leq \beta_{2}
$$

\section{Remainder of the Proof of Proposition 3.9}

The derivation of equilibrium $I I I b$ follows the same lines as the derivation of equilibrium III $a$, the only difference is that $\mu=0$ and consequently $r_{0} \geq$ 1. The equilibrium expressions for $\alpha, F_{0}(p)$ and $F_{1}(p)$ are the same as the expressions for equilibrium III $a$ with $\mu=0$. The restrictions needed for this

\footnotetext{
${ }^{16}$ Since the assumption $\underline{p}_{0}<\underline{p}_{1}$ requires $r_{0}<\frac{2 A}{1-2 \gamma}$, we only need the behavior for $r_{0}<\frac{2 A}{1-2 \gamma}$. It is however interesting to look at the general behavior. It turns out that $g\left(r_{0}\right)=r_{0}\left(1+\frac{1}{2} \ln \left(\frac{r_{0}}{A}(1-\gamma)-1\right)-\frac{1-\gamma}{2 \gamma} \ln \left(\frac{r_{0}}{A} \gamma+1\right)\right)$ is a parabola, such that for each $c$ small enough there are two solutions for $g\left(r_{0}\right)=c$. The smallest solution is the solution we look at and this one is stable. The other solution is unstable, and gives $r_{0}>\frac{2 A}{1-2 \gamma}$. To analyze the stability of a solution, suppose $g\left(r_{0}\right)<c$. This tells that the expected gain from searching when the already obtained price(s) equal $r_{0}$ is lower than the search costs and so consumers will also stop searching when they have obtained a price slightly higher than $r_{0}$. This induces firms to raise their price and so $r_{0}$ increases. The case of $g\left(r_{0}\right)>c$ is reverse and leads to a decrease in $r_{0}$. The smallest solution is in a region where $g\left(r_{0}\right)$ increases, and so is stable. The other solution is in a region where $g\left(r_{0}\right)$ decreases, and so is unstable.
} 
equilibrium to hold are as follows. Note that $\mu=0$ requires $\int_{\underline{p}_{0}}^{1} F_{0}(p) d p<c$ and this gives

$$
c>1+((1-\gamma)-A) \ln \frac{(1-\gamma)^{2}-(1-\gamma) A}{(1-\gamma)^{2}+\gamma A} .
$$

Note that for this restriction to hold, $A$ should be smaller than $1-\gamma$, so $\alpha$ as defined by $\alpha=\frac{(1-\gamma)^{2}-(1-\gamma) A}{(1-\gamma)^{2}+\gamma A}$ is between 0 and 1 whenever the above restriction holds.

\section{Proof of Theorem 4.1}

a Observe that when $c \rightarrow 0$, the equilibrium is given by equilibrium $I b$. This equilibrium has $\alpha=0$ and $\mu=1$. Furthermore, $r_{0}=\frac{c}{\beta_{1}} \rightarrow 0$, while the maximum price equals $r_{0}$.

b Observe that when $A \rightarrow 0$, either equilibrium IIaii or equilibrium IIbii holds.

First look to equilibrium IIaii. In this equilibrium, $\mu$ does not depend on $A, \alpha=1-\frac{A}{(1-\gamma)\left(1-\frac{1}{2} \mu\right)} \rightarrow 1$ and $E p_{1}=A\left(\frac{\frac{1}{2} \mu-\gamma}{\alpha(1-\gamma)\left(1-\frac{1}{2} \mu\right)} \ln \frac{\mu-2 \gamma}{\mu}+\right.$ $\left.\frac{1}{\alpha\left(1-\frac{1}{2} \mu\right)} \ln \frac{1-\frac{1}{2} \mu}{A}\right) \rightarrow 0$.

In equilibrium IIbii, $\alpha=1-\frac{2 A}{(1-\gamma) r_{0}}$, while $r_{0}$ does not depend on $A$. This leads us to $\alpha \rightarrow 1$. Furthermore, $E p_{1}=A \frac{2}{\alpha} \ln \frac{(1-2 \gamma) r_{0}}{2 A}-$ $\frac{A}{(1-\gamma) \alpha} \ln (1-2 \gamma) \rightarrow 0$.

c First note that when $\gamma \rightarrow 0, \beta_{1} \rightarrow 0, \beta_{2} \rightarrow 0$ and $1+\frac{1-\gamma-A}{\gamma} \ln \frac{1-\gamma-A}{1-A} \rightarrow 0$.

This implies that equilibria $I a, I b$ and $I I b$ disappear, so equilibrium II $a$, III a or IIIb holds.

Let us first look at equilibrium IIai. We will rely heavily on the derivation of Proposition 3.8, using the same notation. Whenever $A<\frac{1-2 \gamma}{2}$, equilibrium IIai holds when $g\left(x_{1}\right)<c<g(2 \gamma)$. Note that $g\left(x_{1}\right)<c$ can be rewritten as $\mu<1+\gamma-A-\sqrt{(1+\gamma-A)^{2}-4 \gamma}$ and since $1+\gamma-A-\sqrt{(1+\gamma-A)^{2}-4 \gamma} \rightarrow 0$ equilibrium IIai requires $\mu \rightarrow 0$ when $A<\frac{1-2 \gamma}{2}$. When $\frac{1-2 \gamma}{2}<A<\frac{(1-\gamma)^{2}}{(1+\sqrt{\gamma})^{2}}$, equilibrium IIai holds when $g\left(x_{1}\right)<c<g(2 \gamma)$ or $\max \left\{g(1), g\left(2-\frac{2 A}{1-\gamma}\right)\right\}<c<g\left(x_{2}\right){ }^{17}$ Since $g\left(x_{2}\right) \rightarrow 0$ for $\gamma \rightarrow 0$ the last restriction vanishes. This again leaves the restriction $g\left(x_{1}\right)<c$ or $\mu \rightarrow 0$. The case where $A>\frac{(1-\gamma)^{2}}{(1+\sqrt{\gamma})^{2}}$ need not be considered since $\frac{(1-\gamma)^{2}}{(1+\sqrt{\gamma})^{2}} \rightarrow 1$. We now know that in

\footnotetext{
${ }^{17}$ Note that we only look at the case where $\gamma<\frac{1}{4}$. We can leave the case where $\gamma>\frac{1}{4}$ aside since we are only interested in the equilibrium behavior for $\gamma$ small.
} 


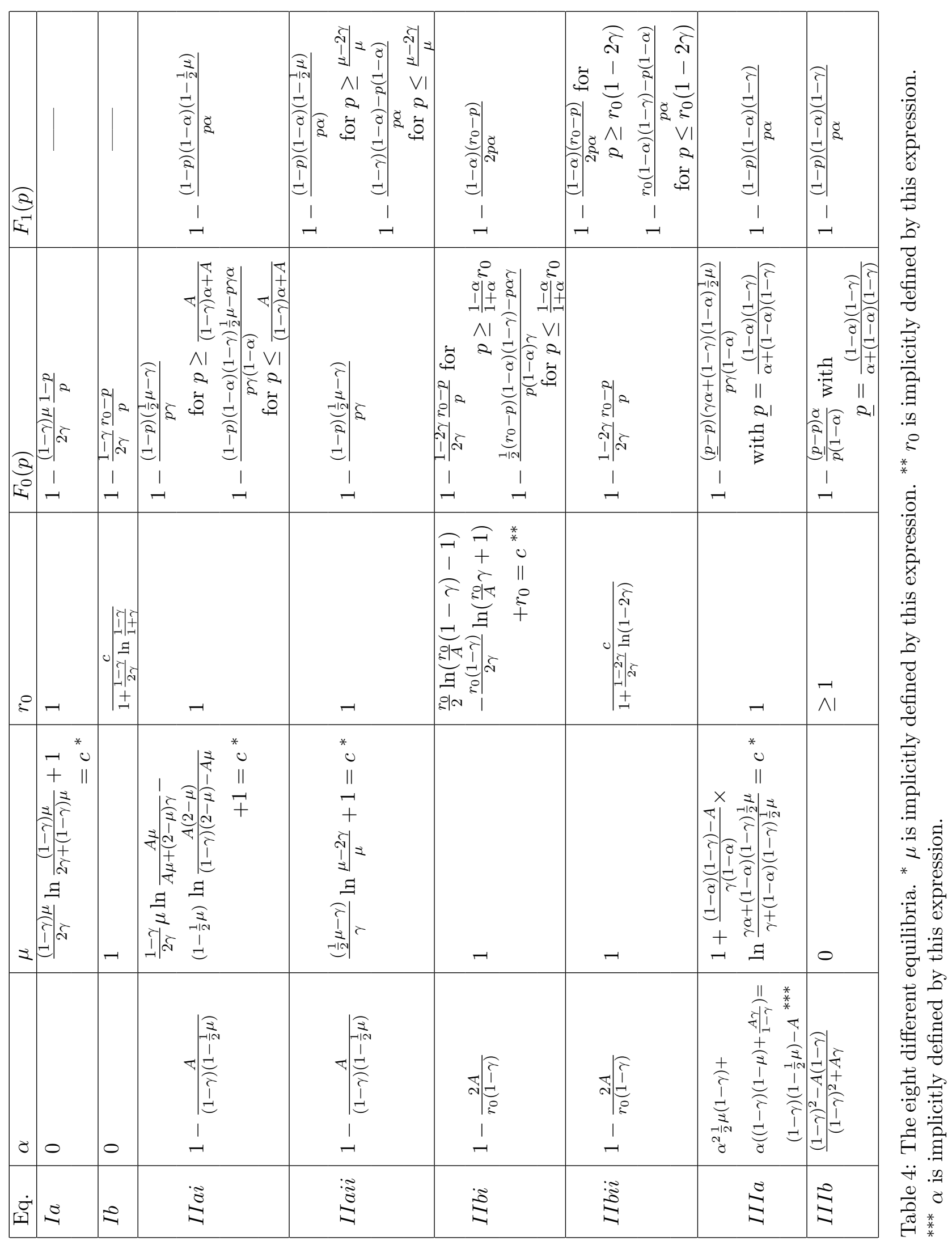


equilibrium IIai $\mu \rightarrow 0$. Using this it is easy to see that $\alpha \rightarrow 1-A$ and $F_{1}(p) \rightarrow 1-\frac{A(1-p)}{p(1-A)}$.

Let us now look at equilibrium IIaii. Note that $1+\frac{\frac{1}{2} \mu-\gamma}{\gamma} \ln \frac{\mu-2 \gamma}{\mu} \rightarrow 0$ for $\gamma \rightarrow 0$ and $\mu>2 \gamma$. Since in equilibrium IIaii $\mu$ is defined by $1+\frac{\frac{1}{2} \mu-\gamma}{\gamma} \ln \frac{\mu-2 \gamma}{\mu}=c$ we conclude that $\mu \rightarrow 2 \gamma \rightarrow 0$. Plugging this in the expression for $\alpha$ gives $\alpha \rightarrow 1-A$ and this finally gives $F_{1}(p) \rightarrow$ $1-\frac{A(1-p)}{p(1-A)}$.

For equilibrium III $a$ to hold, $\mu<2 \gamma$ and so $\mu \rightarrow 0$. Plugging $\mu \rightarrow 0$ and $\gamma \rightarrow 0$ in equation (15) gives $\alpha \rightarrow 1-A$. Plugging $\alpha \rightarrow 1-A$ and $\gamma \rightarrow 0$ in the expression for $F_{1}(p)$ gives $F_{1}(p) \rightarrow 1-\frac{A(1-p)}{p(1-A)}$. For equilibrium $I I I b, \mu=0, \alpha \rightarrow 1-A$ and $F_{1}(p) \rightarrow 1-\frac{A(1-p)}{p(1-A)}$.

\section{Derivation of the comparative statics results in the tables 1 to 3}

The results in the tables are derived by first deriving the derivatives of $\alpha, \mu, E p_{0}, E p_{1}, \pi$ and welfare with respect to $c, A$ and $\gamma$, and then evaluating the sign of these derivatives. We first note that for every equilibrium $\alpha$ and $\mu$ are already defined in the text. Furthermore, for all equilibria except equilibrium $I I I b$ with $\mu=0, E p_{0}=r_{0}-c$. This is by definition since $r_{0}$ is defined as $\int_{\underline{p}_{0}}^{r_{0}}\left(r_{0}-p\right) d F_{0}(p)=c$ or, since $\bar{p}_{0} \leq r_{0}, r_{0}-E p_{0}=c$. In equilibrium $I I I b, E p_{0}=\int_{\underline{p}_{0}}^{\bar{p}_{0}} p d F_{0}(p)$. In all equilibria, $E p_{1}=\int_{\underline{p}_{1}}^{\bar{p}_{1}} p d F_{1}(p)$ and $\pi$ and welfare are as defined in the text.

In some equilibria, several parameters like $\mu$ and $\alpha$ are only implicitly defined. This complicates the derivation of the derivatives. Another complication is that many derivatives have a complicated expression, making it difficult to evaluate the sign. We will now derive the comparative statics results with respect to $c, A$ and $\gamma$ for equilibria $I a$ and $I I b$, thereby showing several of the techniques we also use for the other equilibria. The derivation of the results for the other equilibria is available on request.

In equilibrium $I a, \alpha=0$, so the derivative of $\alpha$ w.r.t. $c, A$ or $\gamma$ is 0 . Define $f(\mu ; \gamma)=\frac{(1-\gamma) \mu}{2 \gamma} \ln \frac{(1-\gamma) \mu}{2 \gamma+(1-\gamma) \mu}+1$. Note that $f(\mu ; \gamma)=c$ implicitly defines $\mu$ as function of $\gamma$ and $c$. Therefore, $\frac{\partial \mu}{\partial A}=0$. Simple calculus gives $\frac{\partial f(\mu ; \gamma)}{\partial \mu}=\frac{(1-\gamma)}{2 \gamma} \ln \frac{(1-\gamma) \mu}{2 \gamma+(1-\gamma) \mu}+\frac{(1-\gamma)}{2 \gamma+(1-\gamma) \mu}$. Since the second derivative of $f(\mu ; \gamma)$ w.r.t. $\mu$ is positive, and $\frac{\partial f(\mu ; \gamma)}{\partial \mu}$ is negative at $\mu=1, \frac{\partial f(\mu ; \gamma)}{\partial \mu}<0$. We also calculate $\frac{\partial f(\mu ; \gamma)}{\gamma}=\frac{-\mu}{2 \gamma^{2}} \ln \frac{(1-\gamma) \mu}{2 \gamma+(1-\gamma) \mu}-\frac{\mu}{2 \gamma+(1-\gamma) \mu}>0$, where the sign of the last expression is determined by noting that the derivative w.r.t. $\mu$ is negative and that at $\mu=1$ the expression is positive. Using that 
$\frac{\partial f(\mu ; \gamma)}{\partial c}+\frac{\partial f(\mu ; \gamma)}{\partial \mu} \frac{\partial \mu}{\partial c}=1, \frac{\partial f(\mu ; \gamma)}{\partial c}=0$ and $\frac{\partial f(\mu ; \gamma)}{\partial \gamma}+\frac{\partial f(\mu ; \gamma)}{\partial \mu} \frac{\partial \mu}{\partial \gamma}=0$, we derive that $\frac{\partial \mu}{\partial c}<0$ and $\frac{\partial \mu}{\partial \gamma}>0$.

In equilibrium $I a, E p_{0}=1-c$ and so $\frac{\partial E p_{0}}{\partial A}=\frac{\partial E p_{0}}{\partial \gamma}=0$ and $\frac{\partial E p_{0}}{\partial c}=-1<$ 0 . We also note that since in equilibrium $I a \alpha=0, E p_{1}$ is not defined. Furthermore, $\pi=\frac{1}{2}(1-\gamma) \mu$. This gives $\frac{\partial \pi}{\partial A}=0$ and $\frac{\partial \pi}{\partial c}=\frac{1}{2}(1-\gamma) \frac{\partial \mu}{\partial c}<0$. We also get $\frac{\partial \pi}{\partial \gamma}=-\frac{1}{2} \mu+\frac{1}{2}(1-\gamma) \frac{\partial \mu}{\partial \gamma}$. To evaluate this expression we use $\frac{\partial \mu}{\partial \gamma}=-\frac{\partial f(\mu ; \gamma)}{\partial \gamma} / \frac{\partial f(\mu ; \gamma)}{\partial \mu} ;$ this gives $\frac{\partial \pi}{\partial \gamma}=\frac{1}{2}\left(\frac{1-\gamma}{\gamma} \frac{(1-\gamma) \mu}{2 \gamma} \ln \frac{(1-\gamma) \mu}{2 \gamma+(1-\gamma) \mu}\right) / \frac{\partial f(\mu ; \gamma)}{\partial \mu}>$ 0 .

Welfare is defined as $W \equiv 1-(1-\gamma) \mu c-(1-\gamma)(1-\mu)$. Note that $\frac{\partial W}{\partial A}=0$, $\frac{\partial W}{\partial c}=-(1-\gamma) \mu+(1-\gamma)(1-c) \frac{\partial \mu}{\partial c}<0$ and $\frac{\partial W}{\partial \gamma}=\mu c+(1-\mu)+(1-\gamma)(1-$ c) $\frac{\partial \mu}{\partial \gamma}>0$.

In equilibrium $I I b, \mu=1$ and so the derivative of $\mu$ w.r.t. the model parameters is 0 . Before deriving the other derivatives, we first take a better look at $r_{0}$, as $r_{0}$ is contained in many expressions. In equilibrium $I I b i, r_{0}$ is implicitly defined by $f\left(r_{0} ; A, \gamma\right) \equiv r_{0}\left(1+\frac{1}{2} \ln \left(\frac{r_{0}}{A}(1-\gamma)-1\right)-\right.$ $\left.\frac{1-\gamma}{2 \gamma} \ln \left(\frac{r_{0}}{A} \gamma+1\right)\right)=c$. Using simple calculus, we derive that $\frac{\partial f\left(r_{0} ; A, \gamma\right)}{\partial r_{0}}=$ $\frac{f\left(r_{0} ; A, \gamma\right)}{r_{0}}+\frac{r_{0}(1-\gamma)}{2} \frac{2 A+r_{0}(2 \gamma-1)}{\left(r_{0}(1-\gamma)-A\right)\left(r_{0} \gamma+A\right)}>0$ since $\frac{2 A}{1-\gamma}<r_{0}<\frac{2 A}{1-2 \gamma}$. Also, $\frac{\partial f\left(r_{0} ; A, \gamma\right)}{\partial A}=\frac{r_{0}^{2}(1-\gamma)}{2 A} \frac{r_{0}(1-2 \gamma)-2 A}{\left(r_{0}(1-\gamma)-A\right)\left(r_{0} \gamma+A\right)}<0$ since $r_{0}<\frac{2 A}{1-2 \gamma}$. These results give $\frac{\partial r_{0}}{\partial c}>0$ and $\frac{\partial r_{0}}{\partial A}>0$. The sign of $\frac{\partial r_{0}}{\partial \gamma}$ depends on the sign of $\frac{\partial f\left(r_{0} ; A, \gamma\right)}{\partial \gamma}=\frac{-r_{0}^{2}}{2\left(r_{0}(1-\gamma)-A\right)}-\frac{r_{0}^{2}(1-\gamma)}{2 \gamma\left(r_{0} \gamma+A\right)}+\frac{r_{0}}{2 \gamma^{2}} \ln \left(\frac{r_{0}}{A} \gamma+1\right)$. This expression can be both positive and negative: when $r_{0}=\frac{2 A}{1-\gamma}, \frac{\partial f\left(r_{0} ; A, \gamma\right)}{\partial \gamma}=A\left(\frac{-2}{(1-\gamma)^{2}}-\right.$ $\left.\frac{2}{\gamma(1+\gamma)}+\frac{1}{\gamma^{2}(1-\gamma)} \ln \frac{1+\gamma}{1-\gamma}\right)$, which is decreasing in $\gamma$ and 0 at $\gamma \approx 0.372$.

In equilibrium IIbii, $r_{0}=\frac{c}{\beta_{2}}$ and so $\frac{\partial r_{0}}{\partial c}=\frac{1}{\beta_{2}}>0, \frac{\partial r_{0}}{\partial A}=0$ and $\frac{\partial r_{0}}{\partial \gamma}=$ $\frac{c}{\gamma \beta_{2}^{2}}\left(\frac{1}{2 \gamma} \ln (1-2 \gamma)+1\right)<0$.

We are now ready to look at the comparative statics results for the advertising intensity $\alpha$, which is given by $1-\frac{2 A}{r_{0}(1-\gamma)}$. First, $\frac{\partial \alpha}{\partial c}=\frac{2 A}{1-\gamma} \frac{1}{r_{0}^{2}} \frac{\partial r_{0}}{\partial c}>$ 0 . Furthermore, $\frac{\partial \alpha}{\partial A}=\frac{2}{r_{0}(1-\gamma)}\left(-1+\frac{A}{r_{0}} \frac{\partial r_{0}}{\partial A}\right)$. In equilibrium IIbi, $\frac{\partial r_{0}}{\partial A}=$ $-\frac{\partial f\left(r_{0} ; A, \gamma\right)}{\partial A} / \frac{\partial f\left(r_{0} ; A, \gamma\right)}{\partial r_{0}}$ and substituting this expression we get $\frac{A}{r_{0}} \frac{\partial r_{0}}{\partial A}-1=$ $\frac{-\left(r_{0} \gamma+A\right)\left(r_{0}(1-\gamma)-A\right) 2 f\left(r_{0} ; A, \gamma\right)}{\left(r_{0} \gamma+A\right)\left(r_{0}(1-\gamma)-A\right) 2 f\left(r_{0} ; A, \gamma\right)+r_{0}^{2}(1-\gamma)\left(2 A-r_{0}(1-2 \gamma)\right)}<0$, so $\frac{\partial \alpha}{\partial A}<0$. In equilibrium IIbii, $\frac{\partial r_{0}}{\partial A}=0$ and so $\frac{\partial \alpha}{\partial A}<0$.

Finally, we look at $\frac{\partial \alpha}{\partial \gamma}=\frac{2 A}{r_{0}(1-\gamma)}\left(-\frac{1}{1-\gamma}+\frac{1}{r_{0}} \frac{\partial r_{0}}{\partial \gamma}\right)$. In equilibrium IIbii, $\frac{\partial r_{0}}{\partial \gamma}<0$ and so $\frac{\partial \alpha}{\partial \gamma}<0$. In equilibrium $I I b i$, we use $\frac{\partial r_{0}}{\partial \gamma}=-\frac{\partial f\left(r_{0} ; A, \gamma\right)}{\partial \gamma} / \frac{\partial f\left(r_{0} ; A, \gamma\right)}{\partial r_{0}}$. A tedious derivation then shows that $-\frac{1}{1-\gamma}+\frac{1}{r_{0}} \frac{\partial r_{0}}{\partial \gamma}<0$ and so $\frac{\partial \alpha}{\partial \gamma}<0$. In equilibrium $I I b, E p_{0}=r_{0}-c$ and so $\frac{\partial E p_{0}}{\partial A}=\frac{\partial r_{0}}{\partial A}$, which is positive in equilibrium IIbi and 0 in equilibrium IIbii. Also, $\frac{\partial E p_{0}}{\partial \gamma}=\frac{\partial r_{0}}{\partial \gamma}$, which 
can be both positive and negative in equilibrium $I I b i$ and is negative in equilibrium IIbii. Finally, $\frac{\partial E p_{0}}{\partial c}=\frac{\partial r_{0}}{\partial c}-1$. For equilibrium $I I b i$, we use that $\frac{\partial f\left(r_{0} ; A, \gamma\right)}{\partial r_{0}} \frac{\partial r_{0}}{\partial c}=1$. Plugging in $r_{0}=\frac{2 A}{1-\gamma}$ shows that $\frac{\partial E p_{0}}{\partial c}$ can be both positive and negative, since $\frac{\partial E p_{0}}{\partial c}$ at $r_{0}=\frac{2 A}{1-\gamma}$ is increasing in $\gamma$ and 0 at $\gamma \approx 0.4345$. In equilibrium IIbii, $\frac{\partial E p_{0}}{\partial c}=\frac{1}{\beta_{2}}-1>0$.

To calculate the derivatives of $E p_{1}$, we first need an expression for $E p_{1}=$ $\int_{\underline{p}_{1}}^{\bar{p}_{1}} p d F_{1}(p)$. In equilibrium $I I b i, E p_{1}=\frac{A}{(1-\gamma) \alpha} \ln \frac{1+\alpha}{1-\alpha}$, while in equilibrium IIbii $E p_{1}=\frac{2 A}{\alpha} \ln \frac{1-2 \gamma}{(1-\alpha)(1-\gamma)}-\frac{A}{(1-\gamma) \alpha} \ln (1-2 \gamma)$. In both equilibria, $\frac{\partial E p_{1}}{\partial \alpha}>0$ (in equilibrium $I I b i$, this is easy to see, in equilibrium IIbii use that $r_{0}>$ $\left.\frac{2 A}{1-2 \gamma}\right)$. This shows that $\frac{\partial E p_{1}}{\partial c}=\frac{\partial E p_{1}}{\partial \alpha} \frac{\partial \alpha}{\partial c}>0$. We now look at $\frac{\partial E p_{1}}{\partial A}$. In equilibrium $I I b i, \frac{\partial E p_{1}}{\partial A}=\frac{1}{(1-\gamma) \alpha} \ln \frac{1+\alpha}{1-\alpha}+\frac{\partial E p_{1}}{\partial \alpha} \frac{\partial \alpha}{\partial A}=\frac{1}{(1-\gamma) \alpha} \ln \frac{1+\alpha}{1-\alpha}-\frac{2}{r_{0}(1-\gamma)} \frac{\partial E p_{1}}{\partial \alpha}+$ $\frac{2 A}{(1-\gamma) r_{0}^{2}} \frac{\partial E p_{1}}{\partial \alpha} \frac{\partial r_{0}}{\partial A}>0$ since some calculus shows that $\frac{1}{(1-\gamma) \alpha} \ln \frac{1+\alpha}{1-\alpha}-\frac{2}{r_{0}(1-\gamma)} \frac{\partial E p_{1}}{\partial \alpha}>$ 0 and it is easy to see that $\frac{2 A}{(1-\gamma) r_{0}^{2}} \frac{\partial E p_{1}}{\partial \alpha} \frac{\partial r_{0}}{\partial A}>0$. In equilibrium IIbii, some calculus shows that $\frac{\partial E p_{1}}{\partial A}=\frac{1}{\alpha^{2}}\left(\frac{1-2 \gamma}{1-\gamma} \ln (1-2 \gamma)-2 \ln \frac{2 A}{r_{0}}-2+\frac{4 A}{r_{0}(1-\gamma)}\right)>0$ since this expression is increasing in $r_{0}$ and $r_{0}>\frac{2 A}{1-2 \gamma}$. The derivative of $E p_{1}$ w.r.t. $\gamma$ gives a very complicated expression. We can however show that for instance in equilibrium type IIbii for $\gamma=0.4$ the derivative is negative for $\frac{A}{c}<0.15096$ and positive for $\frac{A}{c}>0.15096$. This is enough to prove that the derivative can be both positive and negative.

Using the definition of $\alpha$, it is easy to see that $\pi=A$ and so the results in the tables follow.

Finally, we look at welfare. In this case, $W=1-2 A+\frac{4 A^{2}}{r_{0}(1-\gamma)}\left(1-\frac{c}{r_{0}}\right)$. Note that in equilibrium IIbii $\frac{c}{r_{0}}=\beta_{2}$. In equilibrium $\operatorname{IIbi} \frac{\partial W}{\partial c}=-\frac{4 A^{2}}{r_{0}^{2}(1-\gamma)}+$ $\frac{4 A^{2}}{1-\gamma} \frac{2 c-r_{0}}{r_{0}^{3}} \frac{\partial r_{0}}{\partial c}$, what can be shown to be negative. In equilibrium IIbii, $\frac{\partial W}{\partial c}=$ $-\frac{4 A^{2}}{1-\gamma} \beta_{2}\left(1-\beta_{2}\right) \frac{1}{c^{2}}<0$.

In equilibrium IIbii, the derivative w.r.t. $A, \frac{\partial W}{\partial A}=-2+\frac{8 A}{(1-\gamma) r_{0}}\left(1-\beta_{2}\right)$. At $r_{0}=\frac{2 A}{1-2 \gamma}, \frac{\partial W}{\partial A}$ can be both positive and negative, indicating that in general in equilibrium type $I I b$ the derivative of welfare w.r.t. advertising costs $A$ can be both positive and negative.

The derivative of welfare w.r.t. $\gamma$ in equilibrium IIbii is $\frac{\partial W}{\partial \gamma}=\frac{4 A^{2}}{r_{0}(1-\gamma)^{2}}(1-$ $\left.\frac{c}{r_{0}}\right)+\frac{4 A^{2}}{1-\gamma} \frac{2 c-r_{0}}{r_{0}^{3}} \frac{\partial r_{0}}{\partial \gamma}=\frac{4 A^{2}}{r_{0}(1-\gamma)}\left(\frac{1-\beta_{2}}{1-\gamma}+\frac{2 \beta_{2}-1}{\gamma \beta_{2}}\left(\frac{1}{2 \gamma} \ln (1-2 \gamma)+1\right)\right)$. Note that the first part of this expression, $\frac{4 A^{2}}{r_{0}(1-\gamma)}$, is positive, while the second part is a function of $\gamma$ and can be both positive and negative. This shows that in equilibrium $I I b$ welfare can be both increasing and decreasing in $\gamma$.

Note that in the foregoing discussion we obtained exact results even in cases where the derivative is a quite complicated function. This is because in all cases that have been discussed it was possible to find some numerical values that show that (in at least one of IIbi and IIbii) the derivative can be both 
positive and negative. In equilibria $I I a$ and $I I I a$ we sometimes encounter cases where we are unable to find such numerical values. We solve this by plotting the derivatives, to find whether they possibly are altogether positive or altogether negative. A complication is that the derivatives are functions of three variables, which cannot be plotted as a whole. We solve this by fixing one of the variables, $\gamma$, and then plotting the derivatives as a function of the two remaining variables. We fix $\gamma$ to $0.01,0.05,0.1,0.15$, etc., getting a reasonable idea of the behavior of the derivative. The cases where we use this method are marked with an * in the tables.

We note that equilibrium $I b$ has the same structure as equilibrium $I I b$, and can be analyzed in roughly the same way. Equilibria IIa and IIIa can be analyzed in roughly the same way as equilibrium $I a$. In equilibrium $I I I b$ there are no parameters that are implicitly defined, and so this equilibrium is quite easy to analyze.

\section{References}

[1] J.Yannis Bakos. Reducing buyer search costs: implications for electronic marketplaces. Management Science, 43(12):1676-1692, 1997.

[2] Kenneth Burdett and Kenneth L. Judd. Equilibrium price dispersion. Econometrica, 51(4):955-969, 1983.

[3] Gerard R. Butters. Equilibrium distribution of sales and advertising prices. Review of economic studies, 44(3):465-491, 1977.

[4] Peter A. Diamond. A model of price adjustment. Journal of economic theory, 3:156-168, 1971.

[5] Maarten C.W. Janssen, Jose Luis Moraga-Gonzalez, and Matthijs R. Wildenbeest. A note on costly sequential search and oligopoly pricing, June 20042004.

[6] Dmitri Kuksov. Buyer search costs and endogenous product design. Marketing Science, 23(4):490-499, 2004.

[7] Jacques Robert and Dale O. Stahl, II. Informative price advertising in a sequential search model. Econometrica, 61(3):657-686, 1993.

[8] Dale O. Stahl, II. Oligopolistic pricing with sequential consumer search. American economic review, 79:700-712, 1989.

[9] Dale O. Stahl, II. Oligopolistic pricing and advertising. Journal of economic theory, 64:162-177, 1994. 
[10] Dale O. Stahl, II. Strategic advertising and pricing in e-commerce. In Michael R. Baye, editor, Industrial Organization, Advances in Applied Microeconomics, volume 9 of Industrial Organization, pages 69-100. JAI Press, Amsterdam, 2000. 